\title{
LOS LIBROS DE AJEDREZ EN ESPAÑA (1900-1939): REPERTORIO BIO- BIBLIOGRÁFICO Y ESTUDIO BIBLIOMÉTRICO
}

\author{
Jordi Brasó i Rius* \\ Instituto del Centro de Alto Rendimiento de San Cugat del Vallés
}

\begin{abstract}
Resumen: El objeto del trabajo radica en la descripción y análisis de los libros de ajedrez en España en el período de 1900 a 1939. Los medios utilizados han sido las principales bases de datos de las bibliotecas españolas, juntamente con repertorios bibliográficos de la materia en cuestión. Todo ello se ha contextualizado con fuentes secundarias que permiten ubicar las obras analizadas en el contexto social de la época. Posteriormente, un análisis estadístico bibliométrico sirve para valorar e interpretar cuestiones relacionadas con la autoría, el lugar de edición, las editoriales, los años de mayor circulación de obras o los contenidos específicos mayoritarios de las aportaciones. Los resultados son de utilidad para comprobar que Barcelona y también Madrid encabezaron la impresión de libros. También manifiesta una alta producción española y extranjera en torno a los años 30. Entre otros datos es destacable la función divulgativa que tenían la mayoría de las publicaciones.

Palabras clave: Ajedrez; análisis bibliométrico; bibliografía; bio-bibliografía; deporte; España; siglo XX.
\end{abstract}

Title: CHESS BOOKS IN SPAIN (1900-1939): A BIO-BIBLIOGRAPHIC DIRECTORY AND A BIBLIOMETRIC STUDY.

Abstract: The work presents a description and analysis of chess books in Spain in the period 1900 to 1939. The tools used have been the main database of the Spanish libraries, along with bibliographical indexes of the subject matter. This has been contextualized with secondary sources. This allows us to locate the works analyzed in the social context of the time. Subsequently, a bibliometric statistical analysis used to assess and interpret issues of authorship, place of publication, publishers, years of increased circulation of works or controlling specific contents of the contributions. The results are useful to check that Barcelona and Madrid topped the printing of books. Also it manifests high Spanish and foreign production around the year 30. Other data is remarkable the informative function that had the most publications.

Keywords: Chess; bibliometric study; bibliography; bio-bibliography; sport; Spain; twentieth Century.

\section{INTRODUCCIÓN}

El análisis sobre la producción bibliográfica es un elemento de conocimiento sobre la evolución histórica, además de contribuir a dar rigor científico a los diferentes saberes, elementos significativos en el progreso de la sociedad (Punzano, 1981). Uno de los muchos campos de estudio es la educación física y los deportes, con diferentes aportaciones en materia bibliográfica (Blanco, 1927; Bolado, 2012; Pastor, 1995; 2008; Torrebadella, 2014; Torrebadella y Nomdedeu, 2014; Torrebadella y Olivera, 2012).

En este ámbito deportivo se analizan los libros de ajedrez en España en los inicios del siglo XX (1900-1939) y también, como postulaba Menéndez (1876), se tratan diferentes elementos internos de cada obra.

El ajedrez en España se caracteriza por un período brillante y de máximo apogeo, durante el s. XVI, como en el resto de campos en el ámbito intelectual. En esta época, empieza también la literatura ajedrecística. El ajedrecista Ruy López, quien fue considerado el mejor del mundo, en 1561 publica el Libro de Invención Liberal y Arte de jugar al ajedrez. Después de estos años y hasta el s. XIX el juego quedó relegado a un simple elemento de ocio de las clases altas.

El renacimiento del juego surge, según Paluzíe (1917) en Madrid, a finales de los años treinta e inicios de los cuarenta del s. XVIII, con reuniones de ajedrecistas en salones como en el café Levante, o el café Europa. Estas agrupaciones, también en Barcelona, dieron en torno de los años sesenta los primeros círculos en materia del juego. En Madrid, figuras como Golmayo o Martínez de Carvajal propulsan el ajedrez, aunque, la migración de estos dos ajedrecistas a Cuba, frenó en parte el incremento en materia del juego, y quizá fue un motivo del avance de Barcelona como ciudad propulsora del ajedrez. Surgen en la ciudad condal - del mismo modo que en Madrid- salones y cafés de práctica del juego como la desaparecida Sala Imperio -en 1918- y, por lo tanto, figuras ajedrecísticas como Soler, Riu, Bosch de la Trinxeria, Baquero, Tolosa -quien fue director de la sección de ajedrez del Sportsmen's Club, en 1904 y 1905-, Marín, Capó, Riera, Rinck, Puig, Pin y Soler, Paluzíe. Estas iniciativas desembocan en la creación de los

*jordibrasorius@gmail.com

Recibido: 12-01-2016; aceptado: 10-02-2016.

BRASÓ I RIUS, J. Los libros de ajedrez en España (1900-1939): repertorio bio-bibliográfico y estudio bibliométrico. Anales de Documentación, 2016, vol. 19, nº 1. Disponible en: http://dx.doi.org/10.6018/analesdoc.19.1.247821. 
primeros clubes como el Club de Ajedrez Barcelona (1921) y el Club de Ajedrez Condal (1923), que potenciarán la creación de la Federación Catalana de Ajedrez (1925) y posteriormente la Española (1927).

Es remarcable detallar que este fuerte incremento asociacionista, también sucede en muchas otras disciplinas deportivas. De todas formas, se iba a remolque del resto de Europa (Paluzíe, 1917), donde la formación de clubes y entidades en Francia, Alemania o Inglaterra se consolida ya a finales del s. XIX.

Además del análisis asociacionista, para hacernos una idea sobre las inquietudes de la sociedad de la época, hay que analizar también la producción escrita. En lo que se refiere a las publicaciones periódicas destacan revistas del s. XIX como las barcelonesas Teoría y práctica del ajedrez y Ruy López o la madrileña Revista Internacional de Ajedrez. En el siglo XX hay un incremento de estos medios divulgativos con Els Escacs a Catalunya, El Ajedrez Español, Iberia o Jaque, además de boletines como el del Club de Ajedrez de Barcelona, de la Federación de Ajedrez Catalana o más tarde el de la Federación Española. Habrá además que sumar prensa deportiva que trata también el juego, como Stadium, Los Deportes, El Mundo Deportivo, La Vanguardia o Nuevo Mundo entre muchas otras (Brasó, 2014, Di Felice, 2010; Martínez, 1865). Por lo que hace referencia a los libros, además de los de producción en España, destaca una clara influencia extranjera a partir de las importaciones de diferentes escritos que se traducían de Alemania, Francia, Inglaterra, Méjico, Cuba o Argentina.

Centramos el objeto de estudio del presente trabajo en la elaboración de un repertorio de los libros relacionados con el juego en los inicios del siglo XX (1900-1939) en España. A este repertorio se le ha sumado un análisis crítico de cada libro. Además de incluir datos biográficos de los autores, se ha contextualizado cada obra en la sociedad de la época. Finalmente un análisis estadístico también permite dibujar las figuras más representativas de la época, las editoriales predominantes del momento o la cantidad de libros publicados en los diferentes años entre otros aspectos.

\section{MÉTODO, CRITERIOS DE SELECCIÓN Y ORDENACIÓN Y DESCRIPCIÓN DE LAS FUENTES}

El estudio se ha basado en el análisis de las obras de temática ajedrecística que circulaban por España, entre 1900 y 1939. Por lo tanto se han tratado libros nacionales, pero también extranjeros que eran conocidos en todo el territorio español. Se ha limitado la búsqueda a los libros escritos en castellano, catalán, o en caso contrario, publicados en el territorio español. Por su carácter no divulgativo, los manuscritos no se han tenido en cuenta para el estudio. De todas formas son nombrados cuando se analiza la producción bibliográfica.

La ordenación de los libros es cronológica-nominal. Se detalla el asiento bibliográfico de la siguiente forma:

Año AUTORÍA [NOMBRE COMPLETO, LUGAR, AÑO Y FECHA DE NACIMIENTO Y DEFUNCIÓN]: Título de la obra completo. Localidad: Editorial, imprenta, entidad que publica la obra, año. [descripción física]; ${ }^{1}$ tipología del contenido en las obras.

Las obras se agrupan en diferentes tipologías [Tabla I]. Es pertinente resaltar que un libro puede pertenecer a más de una tipología.

\begin{tabular}{|l|c|}
\hline Tipología de las obras & Código \\
\hline Bibliografías & BL \\
\hline Biografías & BI \\
\hline Conferencias & CO \\
\hline Diccionarios & DI \\
\hline Escuela y ajedrez & EA \\
\hline Históricos & HI \\
\hline Juegos varios & JU \\
\hline Literarias & LI \\
\hline Manuales técnicos & MT \\
\hline Partidas & PA \\
\hline Problemas & PR \\
\hline Reglamentos & RG \\
\hline Simbólicos & SI \\
\hline Torneos & TO \\
\hline
\end{tabular}

Tabla I. Clasificación y código de la tipología de las obras de ajedrez analizadas. 
En la elaboración del repertorio se ha partido de la metodología de Simón y descrita por Díez (2000). Han sido analizados, por lo tanto los repertorios y listas de libros publicados hasta la fecha. Como principales obras de referencias destacan las de Garzón, Alió y Artigas (2012), Paluzíe (1912, 1921, 1943) y Sánchez (1934). A la vez, se han consultado y tratado diferentes escritos: Brasó (2013), Fe (1903), Índice bibliográfico (1933), Índice bibliográfico de obras de fondo y algunas de surtido (1912), Llaverías (1910) y Palau (1923-1945).

Además, los catálogos de las bibliotecas españolas, algunos de ellos digitalizados, han concluido esta búsqueda. Destacan las bases de datos de la Biblioteca Nacional de España, el de la Red de Bibliotecas Universitarias, el de las Bibliotecas Públicas del Estado, o el de la Biblioteca de Cataluña.

Juntamente con la bibliografía, se incluye un estudio bibliométrico con elementos imprescindibles en este tipo de trabajos (Pons, 1898). El análisis se refiere a la producción según la autoría, localidad de producción, cantidad de producción en los diferentes años, editoriales promotoras del juego y tipología de los libros.

Hay que añadir que en la misma línea que Menéndez (1876), el repertorio, además de analizar el contenido bibliográfico y su tratamiento estadístico, reflexionamos sobre las mismas obras en su época, hecho que se detalla a continuación.

\section{ESTUDIO DE LAS OBRAS}

Aunque la publicación de libros era costosa y frecuentemente no rentable, el incremento de obras ajedrecísticas del s. XIX no se detiene. Encontramos en los inicios del s. XX una mayor abundancia de libros que circulaban por España, algunos de ellos publicados en las Américas.

Las obras de tipo técnico, de análisis de partidas y de problemas, son las más numerosas en el repertorio. En 1900 se encuentra la obra anónima Prontuario del juego del ajedrez, que algunos bibliófilos coinciden en citar como autor a Francisco Sierra y Real (Garzón, Alió, Artigas, 2012; Palau, 1923-1945; Paluzíe, 1912, 1943). La obra tiene por objetivo dar a conocer el juego e incitar a la práctica. En ella se incluye el reglamento y los principales movimientos aperturas, defensas, gambitos...-. Aunque no destaque por su valor técnico la obra es un reflejo de la voluntad por promover, divulgar y popularizar el juego a la población. Del mismo modo que aparecía esta tendencia en el siglo pasado, se encuentran obras posteriores divulgativas, normalmente de bajo nivel técnico, pero óptimas para el aprendizaje inicial. Es el caso del tratado práctico de Willemanns (1909), el de Roussell-Parcy (1914), la obra mejicana de Lobato (1914), el peculiar y casi desconocido Llibre per a aprendrer a jugar als escacs en 8 dies, en catalán, o la obra no localizada de las partidas de Alekhine y Bogoljubow, Ajedrez, publicada en Ripoll (Bäbler, 1930; García, 1921; Garzón, Alió y Artigas, 2012). Un caso particular es el Primer libro del ajedrecista de Paluzíe (1917), aunque se diferencia de los anteriores tratados por su alto nivel cualitativo, tanto en materia de juego, como de tratamiento de aspectos históricos, biográficos y asociacionistas. También aparecen otras obras como el primer Reglamento del juego del ajedrez, de la Federación Internacional de Ajedrez (c. 1930) o los libros del Dr. Rey (1934, 1939).

En los inicios de siglo, en La Habana destaca la obra El Ajedrez Magistral, de Andrés Clemente (1900), notable ajedrecista y bibliófilo (Clemente, 1896, 1899). El análisis detallado de partidas, juntamente con la reproducción de artículos en el Fígaro de La Habana o el Diario de la Marina de México muestra la intensidad de la afición en Cuba por el juego. La relación entre estos países del otro lado del Atlántico y España es notoria. Así destacan también otras publicaciones que tuvieron repercusión entre la población española, o al menos entre los ajedrecistas del momento. Destaca así la obra de Sandoval (1906); la Cartilla de Ajedrez (Capablanca, 1913); la publicación del Torneo Internacional de Ajedrez (Capablanca, 1913) celebrado en La Habana; la obra divulgativa de Lobato (1914); las conferencias y el interesante Curso de Ajedrez ${ }^{2}$ de Lasker (1918, 1920); el completo tratado argentino de Pérez Mendoza (1920); las partidas de Alekhine (1929) y Palau (1929); la obra ajedrecístico-literaria de Viaña (1929); la de Gelabert y Martínez (1930) -los cuales citan y están influenciados por las obras de Paluzíe y Clemente-; los extensos volúmenes divulgativos de Grau (1930) -que fue director del Ajedrez Americano, campeón de Argentina y ganador del torneo Sudamericano de 1928-, y su versión reducida y económica con la Cartilla de Ajedrez (1931); el curso de Ricardo Reti (1930a) ampliado en una pequeño suplemento en el mismo año (1930b); la obra de la Federación Argentina, Digesto de Ajedrez (1930) -con el reglamento del juego-; la interesante publicación estratégica de Tartakower (1931) -quién publica en 1936 la obra Ideas modernas en las aperturas de ajedrez $z^{3}$, detallada por el campeón de España de ajedrez, el Dr. Rey (1933); la obra anónima Reglamento y código del juego del ajedrez (1935); el manual para iniciados de Hooper (1935) -basándose en las idea de Philidor, Lasker, Morphy y Paluzíe-, y el peculiar librito del ajedrecístico y humorista Cohn (1938). 
JORDI BRASÓ

En estos inicios son también destacables ediciones de obras de gran repercusión y valor publicadas en su primera edición en el siglo anterior. Es la tercera edición de la obra de Borao (1901), publicada en 1858, o diferentes ediciones del Manual del Ajedrecista de Martín Ricart (1897).

Pero habrá que esperar hasta 1905 para encontrar uno de los mejores y más detallados manuales técnicos de calidad. Es el Manual de Ajedrez de Josep Paluzíe (1905, 1911, 1912), bibliófilo y que comparte lugar en la sociedad barcelonesa junto con ajedrecistas de la época como Baquero, Bosch de la Trinxeria o Carbó. Paluzíe hereda la biblioteca ajedrecística de Josep Tolosa -a quien le dedica su primera parte del Manual-, pero además aporta numerosas obras, organiza torneos, construye numerosos problemas, y recibe premios y elogios (Garzón, Alió y Artigas, 2012). La obra analizada representa un tratado en toda regla -se divide en seis partes: preliminares, estrategia, aperturas, finales, problema y miscelánea-, abarcando diferentes ámbitos: historia, partidas, reglamento, finales de partida, construcciones del juego, variantes, datos biográficos, literatura y ajedrez, escuelas de ajedrez, anécdotas y bibliografía. Es por ello que el manual tuvo multitud de ediciones y tiene un alto valor, tanto en materia ajedrecística, como para contextualizar el juego en la sociedad de la época.

Es remarcable citar que las citas de Paluzíe en su bibliografía ajedrecística -incluida en el Manual- nos ha hecho mantener la obra de Niessel (1908), que aunque no se refiera al ajedrez, si que trata sobre un juego estratégico de mesa. Este afán de promoción del juego, es evidente, como también sucede en sus manuscritos existentes en la Biblioteca de Cataluña, ${ }^{4}$ que tratan entre otros aspectos partidas en publicaciones extranjeras. En esta línea destaca su obra en esperanto, Sesdek sakproblemoj (1909), acorde así con los movimientos promotores de esta lengua y que el autor justificaba diciendo que era de fácil aprendizaje. En relación con dicho ajedrecista y bibliófilo, la publicación del Primer ensayo de bibliografía española de ajedrez (1912), que ya apareció en su Manual de Ajedrez y que después de su muerte se completó con las obras que poseía el autor (Paluzíe, 1943) son trabajos indispensables para el estudio histórico de este deporte -como fue la obra de Garzón, Alió y Artigas (2012) o la elaboración de este artículo-. Además es indispensable citar -aunque no tuvo repercusión en su época- el trabajo que Paluzíe dedica al ajedrecista Valentín Marín (Paluzíe, 1913), experto en ajedrez y especializado en la creación y diseño de problemas. En cambio el Primer libro del ajedrecista (Paluzíe, 1917), obra divulgativa, tuvo una alta repercusión, como lo demuestra el sinfín de ediciones que tuvo. El tratado parte de la obra de Filidor aunque le añade numerosos contenidos como el de las partidas y el tratamiento de la evolución histórica del ajedrez.

Paluzíe también divulgó el juego mediante la edición de la obra póstuma de Tolosa y Carreras (1917). Aparecen diferentes problemas publicados en diferentes periódicos con sus respectivas anotaciones, así como dedicatorias, anécdotas y otros datos.

Otro material técnico digno de mención son las obras del que fue Presidente del Club de Ajedrez Barcelona y de la Federación Catalana d’Escacs, Esteban Puig (1908, 1923), con sus apuntes críticos de los finales de partida de su amigo y colaborador Henri Rinck ${ }^{5}$ y sus conferencias técnicas.

Refiriéndonos a Rinck, publica en España unos problemas en francés, relacionados con los finales de partida y que incluyen una breve información de torneos de la época (Rinck, 1919, 1927, 1950). Los escritos tuvieron repercusión en diferentes periódicos y revistas de la época, como La Vanguardia o La Nova Revista. La aparición de estas obras se explican por el origen francés del autor, su posterior residencia en Badalona, y su integración en la red ajedrecística catalana de la época -donde destacan figuras como Tolosa, Marín, Paluzíe, Soler ${ }^{6}$ o Puig-.

Además de Rinck, la influencia francesa vino también de otros autores. Unos años más tarde Champs d’Or tradujo la obra Manual de Ajedrez, del que fue director de La Stratégie (1908-1940), Henri Delaire (1931). España se nutre como iba sucediendo de conocimientos en materia del juego de diferentes países. Se encuentran otros casos similares. Es el de Los grandes maestros del tablero (Reti, 1932), traducido por Bort-Barbosa; la obra de Marcoff (1933) y la de Znosko-Borovsky (1934). Estos libros tuvieron repercusión en los ajedrecistas del momento en España y en la prensa nacional (Garzón, Alió y Artigas, 2012).

Asimismo, y como caso singular destaca El Raja-Yoga y el Ajedrez (Aguirre, 1928) que, aunque analice técnicamente el juego, trata principalmente el ajedrez como medio para el conocimiento del yoga y su actuación en el sistema nervioso.

Del mismo modo y con intensidad similar de publicación a Paluzíe, aparecen los escritos de Juan Bautista Sánchez Pérez, con las obras El ajedrez de D. Alfonso El Sabio (1929), Nómina de aperturas (1930), Epítome de finales y aperturas (1931), Una clasificación de aperturas de ajedrez (1933), Semilla de ajedrez (1933) y Diccionario ilustrado de ajedrez (1934). Si bien la mayoría de este contenido se refiere a los manuales técnicos -aperturas, finales de 
partida, etc. -, destaca Semilla de ajedrez, por su prólogo y por sus contenidos que tratan el uso del juego en la sociedad y en la enseñanza, transcribiéndose incluso un artículo del pedagogo catalán Vergés, introductor del juego en el colegio (Brasó, 2015). Pero su tratado más completo es el diccionario ilustrado que elaboró, con numeroso material en texto y en imágenes, con citas de ajedrecistas, bibliografía, anécdotas, etc. Si sus obras ya tuvieron repercusión, el diccionario fue valorado notoriamente por la prensa de la época, citándose y analizándose en periódicos como $L a$ Vanguardia, Els Escacs a Catalunya o El Ajedrez Español.

A estas publicaciones, hay que añadir un conjunto de recopilatorios de diferentes torneos. Es el caso del torneo de aficionados de San Sebastián de 1911 -publicación con un fin divulgativo del juego- (Rodríguez, 1912), ${ }^{7}$ el de La Habana (Capablanca, 1913) o el de Barcelona (Puig, 1913). De esta última publicación es destacable la referencia que Esteban Puig hace del éxito del torneo de San Sebastián. Detalla numerosos datos de interés del evento -partidas, estadísticas, aportaciones económicas, puntuaciones, historia, notas biográficas...- de gran repercusión, y que fue jugado en la sala Imperio, como ya sucedió en 1909. Y además de estos torneos son destacables las obras que analizan los eventos en Sitges, Hastings y en Reus (Koltanowski, 1936; Primer torneig internacional d'escacs, 1934; Reus. Torneig Internacional d'escacs, 1936; Torneo de Sitges..., 1935).

Las obras históricas del juego también han sido tratadas. Además de los capítulos dentro de los manuales técnicos, como en las obras de Paluzíe (1905) o Sánchez (1933), destacan un conjunto de libros históricos que entre sus páginas tratan el juego. Es el caso del libro de Solalinde (1922) o de Sánchez (1934), donde se recogen las obras de Alfonso X El Sabio, entre las cuales destaca el Libro de ajedrez, dados y tablas. Pero refiriéndonos a este ámbito histórico, y siguiendo la línea de Brunet (1891) aparece la transcripción del manuscrito árabe de Pareja (1935) -que sería un manual técnico para el aprendizaje-. Además es destacable la obra El ajedrez de D. Alfonso el Sabio (Sánchez, 1929), de la cual Els Escacs a Catalunya ya trató, y que sigue la línea de los análisis alfonsinos.

En al ámbito histórico local Francesc Armengol elabora El Club d'Escacs Barcelona, -que tuvo gran repercusión como en Els Escacs a Catalunya, La Veu de Catalunya, El Ajedrez Español o ¡Jaque!- donde además del tratamiento y explicaciones históricas del club, se trata el asociacionismo del momento. La obra es de gran valor en el sentido que permite hacerse una idea detallada del alto nivel de aficionados por la práctica.

Es destacable mencionar a inicios de siglo, dos obras que utilizan el ajedrez para tratar la moralidad de los hombres. Es la obra editada por Brunet (1900), De les costumes dels homens e dels oficis dels nobles sobre l Joch dels Escachs y la transcrita por Bofarull (1902) Libre de Bones Costumes e dels oficis dels nobles. La primera obra analiza los manuscritos de Cessulis traducida al catalán -se publica la obra existente en la Biblioteca Nacional-. Se ha hecho en este idioma teniendo en cuenta que, aunque hay manuscritos del s. XIV y XV en catalán, desde el descubrimiento de la imprenta, no se había hecho ninguna edición en esta lengua. Pretende el editor suplir esta desigualdad y más cuando "els catalans superàvem a les demés nacions no sols en el joc dels escacs sinó en tots els demés rams del saber” (Brunet, 1900, p. V). La obra tiene un sentido catalanista, recordando el autor que el catalán "era una llengua oficial com les demés” (Brunet, 1900, p. V). También afirma en el prólogo la no utilidad del libro para los jugadores de ajedrez, y la poca valoración del aspecto moral en la sociedad:

en el temps dels frontons, academies de billar, carreres de cavalls i de bicicletes, $i$ altres institucions per l'estil, que són les vies que han de conduir-nos a la regeneració nacional i regional, les moralitats, que són lo esencial de l'obra de Cessulis, si, com vulgarment se diu, no serveixen tant com un pegat en un banc, no sen falta gaire. (Brunet, 1900, p. IV)

Brunet habla también de Cessulis -y de todos los manuscritos existentes en diferentes lenguas-, y entre otros aspectos destaca que la obra tuvo en los pueblos del norte mucha popularidad a causa del bajo nivel ajedrecístico que había -el libro solo detalla la posición y movimiento de las piezas-. En cambio en occidente la obra adquirió popularidad por su carácter moral -y no por su interés técnico, donde había numerosos escritos de más alto nivel-. Es curioso citar la obra una década antes de un ajedrecista como Josep Pin y Soler (1892), analizando también aspectos morales -incluso aparece un artículo en esta línea (Moral del ajedrez, 1852)-. Se podría inferir que los ajedrecistas empezaban a darse cuenta de los valores que habría que tener en cuenta en la población.

Por lo que respecta a la segunda obra transcrita por Borafull (1902) se justifica su publicación en una fecha tan cercana a la edición de Paluzíe (Cessolis, 1900) diciendo que la traducción es más completa.

Se han añadido en el repertorio un conjunto de obras literarias que tienen en su título el término ajedrez, y que muestran que el juego era también tratado en un entorno no ajedrecista. Es el caso de la obra de rivalidad amorosa de Villaespesa (1916); las explicaciones del funcionamiento del autómata de ajedrez, de Edgar Allan Poe (1918); el libro 
de Henry Dupuy-Mazuel (1927); el escrito de Viaña (1929); la novela que relaciona la vida con el ajedrez de Miguel de Unamuno (1933); y la humorística de Cohn (1938).

Finalmente, por su carácter de interés general y divulgativo se han tratado obras generales de diferentes juegos, en las cuales aparece el ajedrez. Estos tratados ejemplifican la tendencia ya explicada para promover el juego, juntamente con los manuales técnicos y también con las pequeñas obras para la posibilidad de la práctica a gran cantidad de público, analfabeto en esta materia. Es el caso del libro de Luis de Bizkaya -citado también por Sánchez (1933)alrededor de la primera década del siglo XX, Los juegos. Juegos de la infancia, de sociedad y de sport. Entre los juegos de adivinanzas, de infancia, o de billar, aparece el ajedrez, donde se explica su origen, sus movimientos y las reglas generales. A esta obra, hay que también citar la de Roussell-Parcy (1914) que además trata el juego de las damas. Posteriormente se encuentra también una pequeña obra de reglamentos que además del ajedrez incluye otros juegos como el mus, el póker, el siete y medio o el tute (Reglamentos admitidos para..., 1933). La publicación es una muestra de que el ajedrez se empezaba a introducir en los entornos de ocio más populares.

\section{LISTADO DE LOS LIBROS DE AJEDREZ (1900-1939)}

1900 ANÓNIMO [SIERRA Y REAL, FRANCISCO]: ${ }^{8}$ Prontuario del juego del ajedrez / 1 a parte. ${ }^{9}$ Sevilla: Est. Tip. De 'El Correo de Andalucía', 1900. [127 p.; 15 cm] MT, RG

CESSOLIS, JACME: De les costums dels homens e dels oficis dels nobles, sobre l Joch dels Escachs / Compost per Frare Jacme Cessulis. Translat de latí. Estampada per primera volta la versió catalana, treta d'un ms. Del

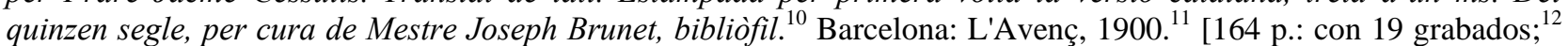
$28 \mathrm{~cm}] \mathrm{SI}$

CLEMENTE VÁZQUEZ, ANDRÉS [1844-1901]: El ajedrez magistral. ${ }^{13}$ La Habana: Imp. del Avisador Comercial, ${ }^{14}$ 1900. [2 v. con 19 diagramas y 3 fotos; $22 \mathrm{~cm}$ ] PA

1902 CESSULIS, JAUME: Libre de bones costumes dels homens e dels oficis dels nobles / Sermó d'en Jaume Cessulis en lati preycat, sobre lo joch dels scachs, trelladat en lo XVen segle a la lenga catalana. Segons un ms. de la Catedral de Girona transcrit per en Manel de Bofarull. ${ }^{15}$ Barcelona: Francisco X. Altés, ${ }^{16} 1902$ [119 p.; 18 cm] SI

1905 PALUZÍE Y LUCENA, JOSÉ [BARCELONA, 1860-1938]: Manual de ajedrez: para uso de los principiantes. Barcelona: Hijos de Paluzíe, ed., 1905-1912. ${ }^{17}$ [3 v. (423 p.+285 p.+347 p.); 23 cm] BI, BL, DC, HI, LI, MT, PA, PR, RG

1906 SANDOVAL, ANDRÉS: Colección de los primeros problemas de Ajedrez publicados por "El País" / y dedicados a los sres. Alain C.White, Henry W. Barry y Dr. Joaquín L. Vallejo, jueces del concurso de problemas abierto por este periódico. ${ }^{18}$ México: Tip. de la Compañía Editorial Católica, ${ }^{19}$ 1906. [107 p.: il. con 50 diagramas; $16,5 \mathrm{~cm}] \mathrm{PR}$

1908 NIESSEL, A. [HENRY ALBERT, FRANCIA, 1866-1955]: Instrucción de los oficiales por medio del Juego de la Guerra / Ejercicios sobre el plano y de cuadros sobre el terreno. Por el capitán _. Breveté de Estado Mayor Francés. Traducido por el Capitán del Regimiento de Infantería Menorca $n .70 R$. Villalba. ${ }^{20}$ Barcelona: Toribio Taberner, ed., ${ }^{21}$ 1908. [91 p; $\left.19 \mathrm{~cm}\right] \mathrm{JU}$

PUIG Y PUIG, Dr. E. [ESTEBAN, BARCELONA, 1878-1940]: Apuntes críticos sobre la obra 150 fins de partie de Mr. Henri Rinck / por el Dr. con una disertación crítica sobre esta clase de composiciones. ${ }^{22}$ Barcelona: Bobes, ${ }^{23}$ 1908. [48 p.; $19 \mathrm{~cm}$ ] PR

1909 WILLEMANNS: ${ }^{24}$ ¿Quiere usted aprender a jugar al ajedrez? / Tratado práctico escrito teniendo a la vista los mejores publicados en España y en el extranjero. Barcelona: F. Granada y Cie, ed., ${ }^{25}$ s.a $1909 .{ }^{26}$ [64 p.: il con un dibujo y dos diagramas; $19 \mathrm{~cm}$ ] DC, MT, RG

PALUZÍE LUCENA, JOZEFO [BARCELONA, 1860-1938]: Sesdek Sakproblemoj / Verkitaj de $\quad .{ }^{27}$ Barcelono: Filoj de Paluzíe, Eldonistoj, 1909. [135 p.: con 60 diagramas; $20 \mathrm{~cm}$ ] PR

1910 BIZKAYA, LUIS DE: Los juegos. Juegos de la infancia, de sociedad y de sport / Naipes, billar, tresillo, ajedrez. Juegos de prendas y adivinanzas. El arte de las gitanas adivinadoras o manera de echar las cartas. Madrid: Aleu, de, s.a. ${ }^{28} 191$ ?..$^{29}$ [124 p.: ${ }^{30}$ il. con un dibujo; $\left.17 \mathrm{~cm}\right] \mathrm{HI}, \mathrm{RG}$

1912 PALUZÍE I LUCENA, JOSÉ [BARCELONA, 1860-1938]: Primer ensayo de bibliografía española de ajedrez. ${ }^{31}$ Barcelona: Hijos de Paluzíe, ed., 1912. ${ }^{32}$ [39 p.: con 5 grabados y un diagrama; 23 cm] BL

RODRÍGUEZ, LUIS [SANTA MARÍA, MADRID, 1881-?]: ${ }^{33}$ Ajedrez. Torneo de aficionados jugado en San Sebastián el mes de marzo de 1911 / Comentarios del Capitán de Artillería D. . Madrid: Imp. del Asilo de Huérfanos del S. C. De Jesús, 1912. [46 p.: con diagramas; 18 cm] TO

1913 CAPABLANCA, JOSÉ RAÚL [LA HABANA, 1888-NEW YORK, 1942]: Cartilla de Ajedrez: para aprender el juego ciencia sin necesidad de maestro: consejos a los principiantes y reglamento universal. ${ }^{34}$ La Habana: Imp. J. A. Casanova, ${ }^{35}$ 1913. [24 p.: con il.; $25 \mathrm{~cm}$ ] MT, RG

CAPABLANCA, JOSÉ RAÚL [LA HABANA, 1888-NEW YORK, 1942]: Torneo Internacional de Ajedrez / celebrado en La Habana del 15 de febrero al 6 de marzo de 1913: colección de las partidas jugadas en el mismo con 
notas analiticas. Por _. ${ }^{36}$ La Habana: Imp. Avisador Comercial, ${ }^{37} 1913$. [118 p.: il. con 54 diagramas y 4 fotos; 24 $\mathrm{cm}] \mathrm{TO}$

PALUZIE I LUCENA, JOSEP [BARCELONA, 1860-1938]: Un Artista en ajedrez: (Valentín Marín). Ensayo crítico / por . . Con la traducción en esperanto por Federico Pujulà y Vallès. Barcelona: Hijos de Paluzíe, ed., ${ }^{38} 1913$. [90 p.: con 27 diagramas y 1 foto; ${ }^{39} 21 \mathrm{~cm}$ ] BI, PR

PUIG Y PUIG, E. [ESTEBAN]: Torneo de ajedrez para el Campeonato de Barcelona 1913 / Su organización, historia i partidas por el Dr.__. ${ }^{40}$ Barcelona: Hijos de Paluzíe ed., ${ }^{41} 1913$. [206 p.. ${ }^{42}$ il. con 1 foto, ${ }^{43} 1$ tabla y 79 diagramas; $20 \mathrm{~cm}]$ TO

1914 LOBATO, J. MANUEL: El contragambito de Greco / Cartillas de ajedrez. Por el profesor_, presidente del club local de ajedrez "Andrés C. Vázquez". ${ }^{44}$ Puebla (México): Antonio Vázquez, ed., ${ }^{45} 1914 .{ }^{46}$ [80 p.: con 11 diagramas; $15 \mathrm{~cm}] \mathrm{MT}$

ROUSSELL-PARCY, JEAN: ${ }^{47}$ ¿Quiere V. jugar al ajedrez? Manual práctico de tan científico juego / formado por _. Barcelona: B. Bauzá ed., s.a. ${ }^{48} 1914 .{ }^{49}$ [80 p.: con 1 dibujo y 28 diagramas; $\left.5016 \mathrm{~cm}\right] \mathrm{MT}$

1916 VILLAESPESA, FRANCISCO [MARTÍN. ALMERÍA, 1877-MADRID, 1936]: Era él. Poema en un acto. La partida de ajedrez. Leyenda dramática en un acto. Madrid: Sociedad de Autores Españoles, ${ }^{51} 1916$. [24 p.; 20 cm] LI

1917 PALUZIE Y LUCENA, JOSÉ [BARCELONA, 1860-1938]: Primer libro del ajedrecista / Contiene nociones elementales y la traducción de la obra de Philidor. ${ }^{52}$ Barcelona: Hijos de Paluzíe, ed., $1917 .{ }^{54}$ [206 p.: il. con 81 diagramas; $20 \mathrm{~cm}$ ] MT

TOLOSA Y CARRERAS, JOSÉ [GERONA, 1826-BARCELONA, 1916]: Problemas de ajedrez / compuestos por _(Obra póstuma). ${ }^{55}$ Barcelona: Hijos de Paluzíe, ed., 1917. [199 p.: con il.; 20 cm] BI, PR

1918 POE, EDGAR ALLAN [BOSTON, 1809-BALTIMORE, 1849]: Nuevas historias extraordinarias. Madrid: Ed. Matev, $1918 .^{56}[218$ p.; $19 \mathrm{~cm}]$ LI

LASKER, E. [EMMANUEL. BERLINCHEN, 1868-NUEVA YORK, 1941]: El sentido común en ajedrez: Resumen de doce conferencias dadas en Londres el año 1895 / Por el Dr.__ (campeón del mundo) y teniendo agregadas al final de la obra las diez y nueve interesantísimas partidas de su match con el sr. W. Steiniz (entonces campeón del mundo), por el cual ganó su título de campeón. Obra traducida del inglés por el Dr. Fructuoso Irigoyen. México: Andrés Botas e hijo, ed., ${ }^{57}$ 1918. [155 p.: con 35 diagramas; $16 \mathrm{~cm}$ ] CO, MT

1919 RINCK, HENRI [LYON, 1870-BADALONA, 1952]: 300 fins partie (3ème édition de "150 fins de partie"). ${ }^{58}$ Barcelona: Hijos de Paluzíe, 1919. ${ }^{59}$ [645 p.: con 313 diagramas; 27 cm] PR

1920 LASKER, E. [EMMANUEL. BERLINCHEN, 1868-NUEVA YORK, 1941]: Curso de ajedrez. / Por el Dr._Campeón del Mundo. Traducido con permiso del autor por el Lic. Agustín Garza Galindo. Tercera Edición. París-México: Librería de la Vda de C. Bouret, ${ }^{60} 1920 .{ }^{61}$ [301 p.: il. con 237 diagramas; 22 cm] MT

PÉREZ MENDOZA, JOSE: El ajedrez en la Argentina. ${ }^{62}$ Buenos Aires: Imp. Tixi y Schaffner, ${ }^{63}$ 1920. [599 p.: con diagramas y fotos; $23 \mathrm{~cm}$ ] HI, RG, LI, BI, TO, EA, CO

1921 GARCÍA BARRAGAN, JOSEP [MÁLAGA, 1893-TERRASSA, 1979]: Llibre per a aprendrer a jugar als escacs en 8 dies / per D. _ . . Primera edició. Terrassa: Imp. La Industria, $1921 .{ }^{64}[11$ p.; $11 \mathrm{~cm}] \mathrm{MT}$

1922 SOLALINDE, ANTONIO G. [GARCÍA. TORO, 1892-MADISON, 1937]: Alfonso X El sabio / Prólogo, selección y glosario. De _. Tomo II. Madrid: Jiménez-Fraud, ed., ${ }^{65} 1922$. [2 v. (275 p. y 233 p.): con un grabado; 16 $\mathrm{cm}$ ] HI, RG

1923 PUIG Y PUIG, DR. E. [ESTEBAN]: La doctrina del final artístico / Resumen de las conferencias dadas en el "Club Barcelona". ${ }^{66}$ Barcelona: Tip. la Académica, s.a. ${ }^{67} 1923 .{ }^{68}[31 \text { p.; } 18 \mathrm{~cm}]^{69} \mathrm{CO}$

1927 DUPUY-MAZUEL, H. [HENRY. 1885-1962]: ${ }^{70}$ El jugador de ajedrez (Novela). ${ }^{71}$ Paris-Madrid: Ed. Españolas, $^{72}$ 1927. [343 p.; $18 \mathrm{~cm}$ ] LI

RINCK, HENRI [LYON, 1870-BADALONA, 1952]: 700 Fins de Partie (4ème édition de "150 Fins de Partie") /

Par $\quad .{ }^{73}$ Barcelona: Tip. la Académica, 1927. [572 p.: ${ }^{74}$ con ilustraciones; $22 \mathrm{~cm}$ ] PR

1928 AGUIRRE Y DE LA TORRE, MANUEL: El Raja-Yoga y el ajedrez. Barcelona: Imp. Clarasó, ${ }^{75} 1928$. [248 p.; $19 \mathrm{~cm}] \mathrm{MT}$, PS

1929 ALEKHINE, ALEJANDRO [MOSCÚ, 1892-ESTORIL, 1946]: Mis mejores partidas de ajedrez 1908-1923 / Por el campeón mundial__ Doctor en Derecho. Traducido del inglés por "PASSER BY". Montevideo (Uruguay): Talleres Gráficos J. Florensa, ${ }^{76}$ 1929. [99 p.; 19 cm] $]^{77}$ BI, PA

PALAU, LUIS [ARGENTINA, 1896-1971]: Combinaciones y celadas en las aperturas. Buenos Aires: Ed. Grabo, ${ }^{78} 1929 .^{79}\left[183\right.$ p.: ${ }^{80}$ con 133 diagramas; $\left.20 \mathrm{~cm}\right] \mathrm{MT}$

VIAÑA, M. [MARIANO]: Trebejos. ${ }^{81}$ Buenos Aires: Grabo, ${ }^{82} 1929$. [187 p.: con 24 diagramas; ${ }^{83} 18$ cm] LI, PR ${ }^{84}$

J. B. S. P. [JUAN BAUTISTA SÁNCHEZ PÉREZ]: El ajedrez de D. Alfonso el Sabio. Madrid: Tip. La Franco Española, ${ }^{85}$ 1929. [121 p.: il. con 112 diagramas, 1 dibujo y 1 foto; $\left.20 \mathrm{~cm}\right]^{86} \mathrm{HI}, \mathrm{PR}$

1930 BÄBLER, JUAN U.: Ajedrez. Ripoll, $1930 .^{87}$ PA, MT

FEDERACIÓN INTERNACIONAL DE AJEDREZ: Reglamento del juego de ajedrez de la Fédération Internationale des Echecs (Federación Internacional de Ajedrez) / Adoptado por la Asamblea general en ocasión del 
Quinto Congreso de la Federación celebrado en la Haya en 1928. Edición oficial española (Derechos reservados). ${ }^{88}$ Valencia: Imp. la Semana Gráfica, c. 1930. [18 p.: con ilustraciones; $21 \mathrm{~cm}$ ] RG

GELABERT, JOSE A. Y MARTÍNEZ, GUMERSINDO: Aperturas de ajedrez / por ex presidente del Club de Ajedrez de La Habana y_ Director del Club de Ajedrez de La Habana. Segunda edición corregida y aumentada. La Habana: Cultural S.A., 1930. [240 p.: con 28 diagramas; 22 cm] MT, RG

GRAU, ROBERTO GABRIEL [BUENOS AIRES, 1900-1944]: ${ }^{89}$ Tratado general de ajedrez. Tomo I. Rudimentos. ${ }^{90}$ Buenos Aires: Grabo, ${ }^{91} 1930 .{ }^{92}$ [215 p.: il.: con 492 diagramas y una foto; $\left.24 \mathrm{~cm}\right]^{93}$ MT

RETI, RICARDO [1889-1929]: Curso superior de ajedrez / Arreglado y corregido por León Colman Lerner. Segunda Edición. Buenos Aires: Lib. de A. Garcia Santos, ${ }^{94} 1930 .{ }^{95}$ [167 p.: con 48 diagramas, 1 grabado y 5 tablas; $19 \mathrm{~cm}]^{96} \mathrm{BI}, \mathrm{MT}^{97}$

RETI, RICARDO [1889-1929]: ${ }^{98}$ Suplemento al Curso Superior de Ajedrez de Ricardo Reti. ${ }^{99}$ Buenos Aires: Lib. de A. Garcia Santos, 1930. [35 p.: con 20 diagramas; $18 \mathrm{~cm}$ ] BI, MT

SÁNCHEZ PÉREZ, J. B. [JUAN BAUTISTA]: Nómina de aperturas / Ajedrez. Primera edición. Madrid: Prensa Moderna Imp., ${ }^{100}$ 1930. [101 p.: con diagramas; $\left.18 \mathrm{~cm}\right]^{101} \mathrm{MT}$

FEDERACIÓN ARGENTINA DE AJEDREZ: Digesto de ajedrez / Contiene: Código Internacional de Ajedrez; Estatutos y Reglamentos de la Federación Argentina de Ajedrez; Estatutos y Reglamentos del Club de Ajedrez Jaque Mate; Fórmulas y "fixtures" para torneos. Apéndice: Reglas del Juego de Damas. ${ }^{102}$ Buenos Aires: Federación Argentina de Ajedrez, s.a. ca. 1930. [86 p.: con tres diagramas; $17,5 \mathrm{~cm}]^{103} \mathrm{RG}$

1931 DELAIRE, ENRIQUE [HENRI, PARÍS, 1860-PARÍS, 1941] ${ }^{104}$ : Manual de ajedrez / Con este manual se puede aprender a jugar perfectamente. Con este manual puede ampliar sus conocimientos el jugador más hábil. Traducción de A. Champs d'Or. Edición ilustrada con209 figuras. ${ }^{105}$ Barcelona: Bauzá, ${ }^{106} 1931 .{ }^{107}$ [121 p.: con 1 dibujo y 224 diagramas; $19 \mathrm{~cm}]^{108}$ MT

GRAU, ROBERTO G. [GABRIEL, BUENOS AIRES, 1900-1944]: Cartilla ajedrez / Por Este libro reúne los elementos necesarios para aprender a jugar al ajedrez sin necesidad de maestros. ${ }^{109}$ Buenos Aires: Grabo, ${ }^{110} 1931 .{ }^{111}$ [88 p.: ${ }^{112}$ il. con 70 diagramas; $\left.17 \mathrm{~cm}\right]$ MT, RG

SÁNCHEZ PÉREZ, J. B. [JUAN BAUTISTA]: Epitome de finales y problemas / Ajedrez. Primera edición. ${ }^{113}$ Madrid: Imp. de Prensa Moderna, 1931. [190 p.: con ilustraciones; 24 cm] MT, PR

TARTAKOWER, DR. SAVIELLY G. [GRIGORIEVICH. ROSTOV, ${ }^{114}$ 1887-PARÍS, 1956]: Sugestiones para la estrategia ajedrecística / Por el _. Traducción del Alemán por Paulino Alles Monasterio. Buenos Aires: Grabo, ${ }^{115}$ 1931. ${ }^{116}\left[96\right.$ p.: ${ }^{117}$ il. con 1 foto, ${ }^{1 \overline{18}} 1$ tabla $^{119}$ y 25 diagramas; $\left.21 \mathrm{~cm}\right]$ MT

1932 RÉTI, RICHARD [PEZINOK, 1889 ${ }^{120}$-PRAGA, 1929]: Los grandes maestros del tablero / Traducción directa del alemán por V. F. Bort-Barbosa. $1^{a}$ Parte. ${ }^{121}$ Barcelona: C. Seither ed., ${ }^{122} 1932 .{ }^{123}$ [181 p.: il. con 1 foto y 38 diagramas; $21 \mathrm{~cm}]^{124} \mathrm{BI}, \mathrm{PA}$

1933 MARCOFF, ALEJO [ALEXIS]: El ajedrez (del método psicológico y de su evolución) / Preparación de celadas, ardides y estratagemas con el fin de desorientar al jugador contrario. Cultivación racional del ingenio y manera de aquilatar la calidad del contrincante. Diferentes formas de ataque y defensa. Edición ilustrada con 27 diagramas. Barcelona: B. Bauzá, ${ }^{125} 1933 .{ }^{126}$ [169 p.: ${ }^{127}$ il. 27 diagramas; 19 cm] MT, PS

SÁNCHEZ PÉREZ, JUAN B. [BAUTISTA]: Una clasificación de aperturas de ajedrez / Mas de 400 maneras de abrir juego. Primera edición. Madrid: Imp. Cleto Vallinas, ${ }^{128} 1933$. [119 p.; $\left.11 \mathrm{~cm}\right] \mathrm{MT}$

SÁNCHEZ PÉREZ, J. B. [JUAN BAUTISTA]: Semilla de ajedrez / Primera edición. Madrid: Imp. Cleto Vallinas, ${ }^{129}$ 1933. [146 p.: il. con 5 fotos, 3 dibujos, 87 diagramas y 1 tabla, $19 \mathrm{~cm}{ }^{130}$ BL, HI, MT, EA

UNAMUNO, MIGUEL DE [BILBAO, 1864-SALAMANCA, 1936]: San Manuel Bueno, Mártir y tres historias más. ${ }^{131}$ Madrid: Espasa Calpe, 1933. [321 p.; $20 \mathrm{~cm}$ ] LI

ANÓNIMO: Reglamentos admitidos por el uso, para los juegos de ajedrez, barracat, julepe, monte, mus, poker, ruleta, siete y media, trenta y cuarenta, tresillo, tute. Madrid: Biblioteca El defensor de Madrid, s.a. ${ }^{132} 1933$. [64 p.: 19 $\mathrm{cm}]^{133} \mathrm{RG}$

1934 ANÓNIMO: Primer torneig internacional d'escacs. Barcelona, Generalitat de Catalunya, 1934. [28 p.: 19 $\mathrm{cm}] \mathrm{TO}$

REY ARDID, Dr. RAMÓN [ZARAGOZA, 1903-1988]: Cien partidas de ajedrez / (1930-1933) extensamente comentadas por el__ campeón de España. Primera edición. Ilustrada con numerosos diagramas. ${ }^{134}$ Zaragoza: Tip. La Académica, ${ }^{135} \overline{1934}$. [266 p.: ${ }^{136}$ il. con 105 diagramas, $22 \mathrm{~cm}$ ] PA

SÁNCHEZ PÉREZ, JUAN B. [JUAN BAUTISTA]: Diccionario ilustrado de ajedrez /: esta obra contiene todos los términos técnicos y vulgares relativos al juego ciencia, con citas de escritores. Primera edición. Madrid: Imp. Cleto Vallinas, ${ }^{137} 1934 .{ }^{138}$ [385 p.: con 45 dibujos, 13 tablas, 5 fotos y 646 diagramas; $\left.23 \mathrm{~cm}\right]^{139} \mathrm{DI}$

ZNOSKO-BOROVSKY, EUGENIO [EVGENII ALEKSANDROVICH. PAVLOVSK (RUSIA), 1884-PARÍS, 1954]: Cómo no debe jugarse al ajedrez / Un método simple claro y lógico de dirigir la partida / Prólogo del Dr. Rey Ardid. Campeón de Ajedrez de España. Versión española de A. Champs D’Or. Edición ilustrada con 14 figuras. ${ }^{140}$ Barcelona: Bauzá, ${ }^{141} 1934 .{ }^{142}$ [125 p.: ${ }^{143}$ il. con 14 diagramas; 19 cm] MT 
1935 ANÓNIMO: Reglamento y código del juego de ajedrez / Contiene: Código del juego. Reglamento de Torneos. Fórmulas para hacer los "Fixtures". Reglas para las Ventajas de Piezas de Tiempos. Sistemas de Anotaciones, Descriptivo y Algebráico. Adoptado por la Federación Argentina de Ajedrez. Buenos Aires: Editorial Grabo, ${ }^{144} 1935 .{ }^{145}$ [32 p.: con 2 dibujos, 4 diagramas y 20 tablas; $\left.18 \mathrm{~cm}\right]^{146} \mathrm{RG}$

ANÓNIMO: Torneo internacional de ajedrez de Sitges año 1934 / Con 18 grabados y 19 diagramas. Barcelona: C. Seither ed., ${ }^{147} 1935$. [71 p.: il. con 18 grabados y 119 diagramas; $21 \mathrm{~cm}$ ] TO

ARMENGOL, F. [FRANCESC 1894-1977]: El Club d'Escacs Barcelona vist per mi / Primera edició. ${ }^{148}$ Barcelona: Pereda, Impressor, ${ }^{149}$ 1935. [208 p.; $\left.{ }^{150} 18 \mathrm{~cm}\right] \mathrm{HI}$

HOOPER KOELTZ, W. ${ }^{151}$ [SANTA CLARA, 1887- BURGOS, 1937]: Manual práctico de ajedrez. Madrid: Ed. Ibéricas, ${ }^{152} 1935 .{ }^{153}$ [189 p.: ${ }^{154}$ il. con 1 dibujo y 91 diagramas; $\left.16 \mathrm{~cm}\right] \mathrm{MT}$

PAREJA CASAÑAS, FÉLIX M. [MARÍA. BARCELONA, 1890- ALCALÁ DE HENARES, 1983]: Libro del ajedrez. De sus problemas y sutilezas. De autor árabe desconocido. Según el Ms. Arab. Add. 7515 (Rich) del museo británico / texto árabe, traducción y estudio previo por $\quad{ }^{155}$ Madrid i Granada: Escuela de estudios árabes de Madrid y Granada. Imp. de Estanislao Maestre, ${ }^{156} 1935 .{ }^{157}$ [2 t. (503 p. + 378 p.): con diagramas; 23 cm] HI, MT

SÁNCHEZ PÉREZ, J. A. [JOSÉ AUGUSTO, 1882-?]:" ${ }^{158}$ Alfonso X el Sabio siglo XIII. Madrid: M. Aguilar Editor, s. a. ${ }^{159} \mathrm{ca}$. $1934-1935 .{ }^{160}$ [299 p.: con 5 il.; $\left.{ }^{161} 16 \mathrm{~cm}\right] \mathrm{HI}$

1936 ANÓNIMO: Reus Torneig internacional d'escacs. Organitzat pel Club d'Escacs Barcelona sota el patronatge de la Generalitat de Catalunya / Ajuntament i escola del treball de Reus. Sota els auspicis de la Federació Catalana d'Escacs. Autoritzat per la Federació Española d'Escacs. Tindrà lloc a l'escola del Treball de Reus del 20 de juny al ler de juliol. Reus, Imp. Cuscó, ${ }^{162}$ 1936. [44 p.: il. con 1 imagen, 21 fotos y un diagrama; $16 \mathrm{~cm}$ ] ${ }^{163} \mathrm{HI}, \mathrm{TO}$

KOLTANOWSKI, G. [GEORGE. AMBERES, 1903-SAN FRANCISCO, 2000]: Torneo Internacional de Hastings: $1935-1936$ / Con 19 diagramas. Barcelona: C. Seither, ed., ${ }^{164} 1936 .{ }^{165}$ [55 p.: il. con 19 diagramas y 3 tablas; $22 \mathrm{~cm}] \mathrm{TO}$

1938 COHN, HANS [BRATISLAVA, 1897-GUATEMALA, 1964]: Lances humorísticos de ajedrez / Por Con 10 ilustraciones conmovedoras y 58 diagramas. Guatemala: Unión Tipográfica, 1938. [52 p.: 58 diagramas y 10 dibujos; $20 \mathrm{~cm}$ ] LI, PR

1939 REY ARDID, DR. RAMÓN [ZARAGOZA, 1903-1988]: Los principios del ajedrez / Por el Dr. Ramón Rey Ardid campeón de España. Primera edición ilustrada con numerosos diagramas. ${ }^{166}$ Zaragoza: Tip. La Académica, ${ }^{167}$ 1939. ${ }^{168}$ [237 p.: ${ }^{169}$ il. con 202 diagramas, $\left.22 \mathrm{~cm}\right] \mathrm{MT}$

\section{ANÁLISIS ESTADÍSTICO DE LAS OBRAS}

Del conjunto de libros se han analizado estadísticamente los siguientes aspectos: autoría, lugar de edición de las obras, cantidad de libros publicados en los diferentes años, editorial encargada de producir la obra, tipología por lo que respecta al contenido de cada obra.

En el caso de la autoría, hay once autores que acumulan el $45 \%$ de todas las obras analizadas. Y dentro de estos productores sobresalen el bibliófilo José Paluzíe y Juan Bautista Sánchez, que acumulan entre ambos el 16\% de la producción.

Por lo que respecta al lugar de edición de los libros, y como sucede con el grado de asociacionismo y actividad ajedrecística, Barcelona -con un 36\% - y en menor medida Madrid -con un 26\%- son las ciudades principales donde se publican los libros que circulan por España. El resto de ciudades españolas sólo suma el 6\% de las obras, lo que indica la polarización ajedrecística en estas dos ciudades. Es digno de mención la cantidad de obras extranjeras que circulaban por España, alrededor del 30\%, hecho que muestra las relaciones tanto transatlánticas como de diferentes países del continente.

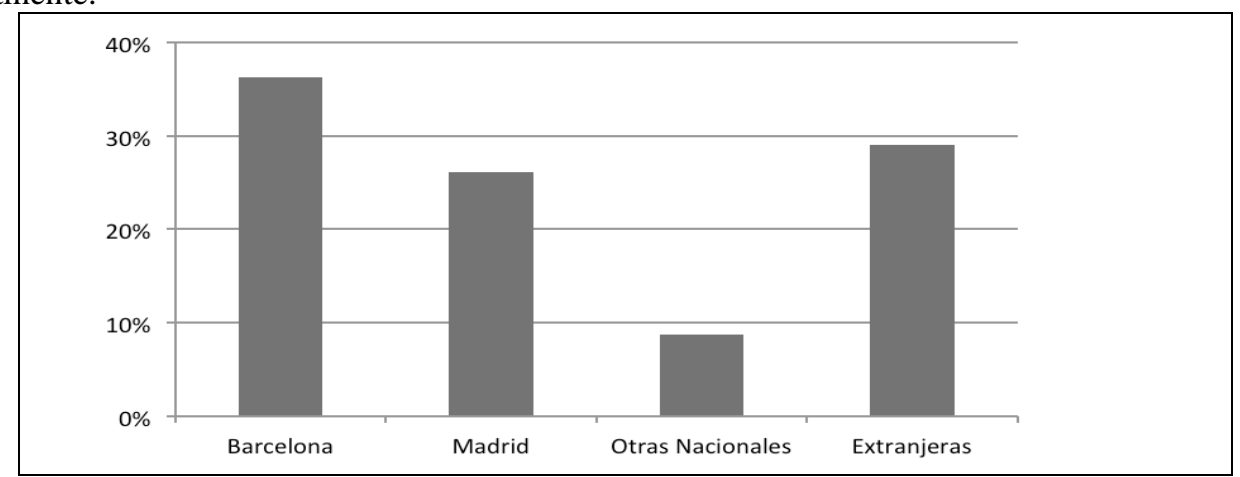

Figura 1. Lugar de publicación de las obras de ajedrez analizadas. 
Por lo que hace referencia a las editoriales en materia de ajedrez, aunque la mayoría publican de forma ocasional, destacan dos editoriales promotoras del juego, como son la de los Hijos de Paluzíe, de Barcelona y la editorial Grabo, de Buenos Aires. Con un 9\% del total de las publicaciones cada una -si computamos el Manual de Paluzíe (1905, 1911, 1912) como tres libros, la editorial llega al $12 \%$ del total de las publicaciones-, es decir alrededor del $20 \%$ del total, representan los principales promotores del juego a través de los libros.

\begin{tabular}{|l|r|c|}
\hline Editorial & Obras & Porcentaje \\
\hline Grabo & 6 & $9 \%$ \\
\hline Hijos de Paluzíe & 6 & $9 \%$ \\
\hline B. Bauzá & 4 & $6 \%$ \\
\hline C. Seither & 3 & $4 \%$ \\
\hline Cleto Vallinas & 3 & $4 \%$ \\
\hline La Académica & 3 & $4 \%$ \\
\hline Otras & 44 & $64 \%$ \\
\hline TOTAL & 69 & $100 \%$ \\
\hline
\end{tabular}

Tabla II. Número y porcentaje de obras de las principales editoriales, entre 1900 y 1939.

La periodicidad de las obras de ajedrez publicadas en los diferentes años es una variable también analizada. Se comprueba que en el período de 1900-1929 la publicación es mínima, con muchos años sin novedades -sí había en cambio, nuevas ediciones de obras antiguas-. Los momentos en que se publicaron obras en estos períodos son como mucho de dos obras por año $-\mathrm{y}$ a menudo representaban publicaciones extranjeras-. Hay que destacar el período de 1929 a 1935, donde se publican el 49\% de las obras totales, momento que coincide con el final de la dictadura de Primo de Rivera y la Segunda República. Este auge queda paralizado con la Guerra Civil, donde la producción de libros es nula hasta finales de este período -sólo el 3\%-.

En el análisis sobre las tipologías de las obras se han agrupado en categorías similares -ver Tabla III-. Hay que tener en cuenta además que un mismo libro puede incluir más de una temática.

\begin{tabular}{|l|l|c|}
\hline Tipología de las obras & Códigos & Porcentaje \\
\hline Material técnico, partidas y problemas & MT, PA, PR & $61 \%$ \\
\hline Reglamentos & RG & $19 \%$ \\
\hline Historia del ajedrez y sus organizaciones & HI & $16 \%$ \\
\hline Biografías y bibliografías & BI, BL & $13 \%$ \\
\hline Torneos & TO & $10 \%$ \\
\hline Literarias & LI & $4 \%$ \\
\hline Diccionarios & DI & $3 \%$ \\
\hline Conferencias & CO & $13 \%$ \\
\hline
\end{tabular}

Tabla III. Aparición de los principales contenidos-tipologías en las obras de ajedrez analizadas.

Se comprueba que el fin principal de la mayoría de las obras era divulgativo para el aprendizaje del juego, mediante manuales técnicos o libros de problemas y partidas -61\%-. En relación con este aspecto, los reglamentos están también presentes, y se publican libros o capítulos específicos destinados a detallar las normas del ajedrez $19 \%-$. Si sumamos las dos tipologías de obras, computan el $80 \%$ del total de las publicaciones que circulaban por España.

Es curioso destacar la voluntad por divulgar el juego en un ámbito más histórico -sobre los orígenes, pero también relacionado con las biografías y las bibliografías- y de información actual de la época de los torneos. Contando estos dos aspectos, equivalen al 30\% del total de las obras. 
Aparecen también libros literarios con una temática ajedrecística -10\%-, hecho que muestra que el juego se iba integrando en la sociedad de la época. Y un último aspecto son los diccionarios y libros de conferencias ajedrecísticas, que aunque ocupen el último lugar en la tipología de las obras son también un ejemplo de la voluntad de promover el juego, ya sea detallando todos los conceptos, o divulgando las ideas y filosofías que los principales ajedrecistas del momento habían detallado en eventos organizados principalmente por las asociaciones y clubes ajedrecísticos de la época.

\section{CONCLUSIONES}

La publicación de obras ajedrecísticas en los inicios del siglo XX (1900-1939) representa una muestra significativa de la cada vez mayor importancia que la sociedad española empezaba a darle al juego. De este modo, a estas obras, le acompañan un conjunto de publicaciones periódicas y boletines en materia ajedrecística que pretenden divulgar tanto la normativa y técnica del juego, como los eventos de interés social que se van produciendo. Estos escritos frecuentemente estaban fomentados por jugadores que pertenecían a diferentes clubes y asociaciones. Aunque destacan figuras como Paluzíe, Tolosa o Sánchez Pérez, numerosos son los autores que tienen por objetivo divulgar el juego desde diferentes enfoques -en la mayoría de los casos por lo que respecta a la técnica del juego y su normativa-.

Como sucede con la mayoría de los deportes, Barcelona, y también Madrid, encabezaban estas inquietudes en materia de ajedrez. Es notorio, recordar que las importaciones de obras extranjeras tuvieron un peso relevante en la divulgación del juego a España.

El repertorio en ningún caso es cerrado, ya que seguramente irán apareciendo obras nuevas. Asimismo el análisis sobre otras publicaciones, ya sean revistas o manuscritos, juntamente con biografías de los principales ajedrecistas del momento es necesario para conformar una imagen completa del ajedrez en la sociedad española.

\section{NOTAS}

${ }^{1}$ Número de páginas, ilustraciones y altura de la cubierta en centímetros.

${ }^{2}$ La obra en parte es una recopilación de artículos publicados por el autor en el Lasker's Chess Magazine entre 1904 y 1908.

${ }^{3}$ Se ha consultado la obra de 1941, publicada en Argentina por la editorial Sopena.

4 Ms. 946, 1352, 1539, Ms. 1540. Garzón, Alió y Artigas (2012) analizan también un manuscrito de Paluzíe: Spanish Chess Flowers (1925), ubicado en la Cleveland Public Library; y el Ms. 1510 de la Biblioteca de la Haya, Some Chess-Problems (1934).

${ }^{5}$ Son dignos de mención los manuscritos citados por Garzón, Alió y Artigas (2012): La doctrina del final artístico -en catalán y en castellano-, que incluyen las conferencias del autor en el Club d'Escacs de Barcelona.

${ }^{6}$ Plácido Soler se educó en la Escuela del Mar, colegio que tenía en su metodología la práctica obligatoria del ajedrez (Brasó y Torrebadella, 2014).

${ }^{7}$ Paluzíe ya reseñó la publicación en La Vanguardia, y la alabó (Paluzie, 1917).

${ }^{8}$ Garzón, Alió y Artigas, 2012; Paluzíe, 1912, 1921, 1943.

${ }^{9}$ No hay constancia de la segunda parte.

${ }^{10}$ El manuscrito que se traduce se encuentra en la Biblioteca Nacional de España.

${ }^{11}$ Impreso el 15 de junio. La editorial Nabu Press en 2011 hace una reproducción. Se vendía a 16 ptas.

12 Garzón, Alió y Artigas (2012) apuntan que los grabados pertenecen a la edición inglesa de Caxton (1474).

13 Hay una dedicatoria, en el ejemplar de la Biblioteca de Cataluña que corresponde a la antigua biblioteca Paluzíe, de Juan Corzo, el 10 de agosto de 1912 a dicho bibliófilo.

14 Ubicación: Amargura, 30.

${ }^{15}$ Manel Bofarull [1816-1892] es el transcriptor.

${ }^{16}$ Ubicación: Carrer dels Àngels, 22 y 24.

17 En tres tomos: 1905 -Tomo I-, 1911 -Tomo II- y 1912 -Tomo III-. Garzón, Alió y Artigas (2012) detallan las posteriores y numerosas ediciones.

${ }^{18}$ Paluzíe (1912, 1921, 1943) cita la obra. Precio de venta: 50 centavos. Es la primera edición, aunque no tenemos constancia de una segunda. En el ejemplar de la Biblioteca de Cataluña, se cita la editorial B. Bauzá, ubicada en la calle Aribau, 175 de Barcelona, y el precio de venta de 2 ptas., hecho que demuestra la relación y transmisión de información entre España y América. En la obra se anuncia el libro ¿Quiere V. Jugar al ajedrez? de Roussell-Parcy (1914).

19 Segunda de San Lorenzo, n. ${ }^{\circ} 19$.

${ }^{20}$ En la bibliografía ajedrecística de Paluzíe (1943) se incluye la obra. Este hecho justifica su inclusión en el catálogo.

${ }^{21}$ Ubicación: Calle Rosellón, 224. Se indica también: Tip. Electra, en el Pasaje de Mercader, 4.

22 Además de una dedicatoria a mano de Puig a Paluzíe, la obra tiene una dedicatoria a Rink.

23 Talleres Gráficos Bobes, en Balmes, 30 y Diputación, 232.

${ }^{24}$ Según Paluzíe, es un seudónimo. Garzón, Alió y Artigas (2012) afirman que el autor podría ser Enrique Flaquer. 
${ }^{25}$ Es la continuadora de las publicaciones Granada. Ubicada en la calle Diputación, 344, de Barcelona y Mesoneros Romanos, 10, en Madrid. La Tip. es C. Gisbert, ubicada en Nueva de San Francisco, 22 de Barcelona. Se vendía a 50 cts. y se cita el n. ${ }^{\circ} 5753$ de la Tip. El Anuario, calle Diputación, 344. F. Granada y Ca.

${ }^{26}$ En el registro de la BC indica que es de 1909 y en el de la BNE que es de 1910. En la parte VI del Manual de Ajedrez de Paluzíe, se comenta que la obra apareció en 1909. Además hay tres ediciones, todas idénticas en su contenido: la primera impresa por la Tip. El Anuario, publicada en Barcelona y Madrid, la segunda sólo en Barcelona (c. 1925) y la tercera, publicada por la ed. Atlante, continuadora de las publicaciones Granada, tal y como se indica en la obra (c. 1927).

27 Obra en esperanto, aunque con traducción en diferentes idiomas.

${ }^{28}$ Ubicada en Valverde 36, bajos. La imprenta es Félix Moliner.

29 Según datos de la BNE. Obra citada por Sánchez (1933).

${ }^{30}$ Las páginas que tratan el ajedrez son de la 79 a la 83.

${ }^{31}$ La tirada de la obra es de 100 ejemplares y tiene el mismo contenido que el capítulo XVI de la parte sexta del Manual de Ajedrez para uso de los principiantes (Paluzíe, 1905-1912, 1921)

32 Otras ediciones: 1921 -donde se añaden obras de 1913 a 1919- y 1927 -donde se añaden obras de 1923 y 1924 -.

33 Según Garzón, Alió y Artigas (2012).

34 Paluzíe (1943) disponía de un ejemplar de la obra.

35 En la calle Compostela n. ${ }^{\circ} 89$.

${ }^{36}$ La obra era conocida en España (Paluzíe 1943). El ejemplar de la BC tiene una dedicatoria de Juan Corzo a Paluzíe -Habana, 28 de noviembre de 1913-. Capablanca dedica la obra al Ayuntamiento de la Habana y a su alcalde, el general Freyre.

${ }^{37}$ En la calle Amargura, 30, Esq. A.

38 Imp. Hijos de Paluzíe.

39 De Valentín Marín.

40 Edición de 150 ejemplares. Se vendía a 5 ptas. Dedicada a los “Señores Protectores del Torneo de Ajedrez celebrado en Barcelona para el Campeonato del año 1913”.

${ }^{41}$ Imp. de la Casa Prov. de Caridad (Barcelona).

42 Las quince páginas iniciales están numeradas en números romanos.

43 Imagen de los participantes y directores del campeonato.

44 Paluzíe (1943) disponía de un ejemplar.

45 Portería de Sta. Clara 3. También se indica: Talleres de Imp. y encuadernación "El Escritorio”.

46 Se terminó de imprimir el 30 de enero. Se comenta que está en prensa la Cartilla del Giuoco Piano, volumen con más de 300 páginas.

47 Es un seudónimo (Paluzíe, 1912).

48 Colección: Biblioteca de Sports y Juegos. El emplazamiento del editor es la calle Aribau, 175 a 179.

49 La referencia ha sido tomada de la BC y de la revista Stadium (De Ajedrez, 1914) en que se informa de la publicación de la obra. En la revista se citan párrafos del libro y se informa que está copiado del Manual de Paluzíe. Se hizo una tercera edición titulada Los primeros pasos en ajedrez (c. 1942) y una versión en catalán en 1986 (Garzón, Alió y Artigas, 2012).

${ }^{50}$ En la cubierta anterior indica 27 grabados, pero se ha incluido un tablero vacío -no computado por el autor-.

51 A cargo de Félix Costa. La imprenta se ubica en la calle Asalto, 40.

${ }^{52}$ La obra de Philidor a la que se refiere es de 1777, el Analyse du jeu des échecs. Según Garzón, Alió y Artigas (2012), Puig ofreció al autor la traducción del manuscrito del tratado de Philidor, pero Paluzíe hizo su propia traducción.

53 A partir de 1927, la imprenta es Elzeviriana y Librería Camí.

54 Aparecen diferentes ediciones: ed. Hijos de Paluzíe (1917, 1918, 1921, 1923); de la imp. Elzeviriana y Librería Camí, ubicada en la calle Torres Amat, 9, -a partir de 1927-. La imprenta y editorial es la "cesionaria única de las producciones de la antigua editorial Paluzíe” y se ubica en la Calle Joaquín Costa, 64 -casa de los editores-. Hay más ediciones: $1931\left(6^{a}\right), 1935\left(7^{\mathrm{a}}\right), 1938\left(8^{\mathrm{a}}\right), 1942\left(9^{\mathrm{a}}\right), 1945\left(10^{\mathrm{a}}\right) \ldots, 1952\left(14^{\mathrm{a}}\right), 1958\left(16^{\mathrm{a}}\right)$. Se anuncian al final de la obra, otros libros del editor, y que se venden en las principales librerías de España y America y en la casa de los editores: de Paluzíe -Manual de ajedrez para uso de principiantes en 3 tomos, Sesdek sakproblemoj (Sesenta problemas de ajedrez) -; de Marín -Un artista en ajedrez-; de Puig -Torneo de ajedrez para el campeonato de Barcelona, 1913-; de Tolosa-Problemas de ajedrez-.

${ }^{55}$ Recopila los problemas de Paluzíe.

56 Aunque la obra es de 1818, El jugador de ajedrez fue un artículo para descubrir el falso autómata. En 1918, Ed. Matvev lo incluye en su obra. En 1921, la librería Fernando Fe lo vuelve a publicar con traducción de Ramón Gómez de las Serna.

${ }^{57}$ Ubicación: $1^{\text {a }}$ Bolívar, n. ${ }^{\circ}$ 9. Se detalla la imprenta Helénica en el Pasaje de la Alhambra, 3. Por lo tanto, la obra se imprimió en España.

${ }^{58}$ Obra en francés pero publicada en Barcelona.

59 Se hicieron 24 ejemplares en “papier japon” y 24 en "papier á la cuve númerotés à la presse”. Hubo ampliaciones de la obra: 700 fins de partie (1927) y 1414 fins de partie (1950).

${ }^{60}$ Ubicación: 23, Rue Visconti en Paris y 45, Cinco de Mayo, en Méjico.

61 Edición notablemente corregida, de las dos ediciones anteriores: 1907 y 1914. Además, esta 3 ${ }^{a}$ edición, tiene una parte nueva -y aun así, la obra sigue incompleta, según se comenta en el prólogo-. Hay ediciones posteriores: $1922\left(4^{\mathrm{a}}\right), 1924\left(5^{\mathrm{a}}\right), 1926\left(6^{\mathrm{a}}\right), 1928\left(7^{\mathrm{a}}\right), 1930\left(8^{\mathrm{a}}\right)$. La obra se cita en Sánchez (1933). La segunda edición se incluye en Paluzíe (1943). Además, en el Índice Bibliográfico (1933) de la librería Sintes, también se indica su venta al precio de 12-15 ptas. -ref. 1097; p. 173-, por cierto, mucho más elevado que las obras publicadas en España, que se vendían entre 3 y 5 ptas.

62 Se incluye en Sánchez (1933) y en Paluzíe (1943). El ejemplar de la BC está dedicado a Paluzíe.

${ }^{63}$ Venezuela, 336, de Buenos Aires.

64 Obra no consultada y listada en Garzón, Alió y Artigas (2012). 
${ }^{65}$ Colección Granada. En el segundo volumen se encuentra el apartado de: "Libros de ajedrez, dados y tablas".

${ }^{66}$ En el ejemplar de la BC hay una dedicatoria a Paluzíe "Al Honorable mestre de la valiosa cooperació en el 1r concurs Nacional de Problemes ‘Els Escacs a Catalunya' li dedica son deixeble i admirador, l’autor” (Barcelona, 19 maig 1929).

${ }^{67}$ De Serra y Rusell.

${ }^{68}$ Según Paluzíe (1927).

${ }^{69}$ En la portada hay el diagrama número 149 de la obra 300 Fins de Partie, de Rinck.

${ }^{70}$ Traducción de J. M. Aguado de la Loma.

${ }^{71}$ Obra original francesa titulada Le joueur d'Échecs.

${ }^{72}$ G. Hernández y Galo Sáez. Colección: Las Grandes Novelas Contemporáneas.

${ }^{73}$ Obra en francés pero publicada en Barcelona.

${ }^{74}$ De las cuales 32 páginas son introductorias.

${ }^{75}$ Ubicación: Villarroel, 17.

${ }^{76}$ Cerrito, 740.

${ }^{77}$ Citada en Bibliografia Ajedrecística (Rey, 1933).

${ }^{78}$ Se informa que es la editora de El Ajedrez Americano.

${ }^{79}$ Aparecen numerosas ediciones.

${ }^{80}$ Se anuncia la revista mensual El Ajedrez Americano.

${ }^{81}$ La obra se conocía en España (Paluzíe, 1943; Sánchez, 1933). Obra dedicada a la madre del autor. Es un homenaje a la Cámara de diputados de la Nación a cuyo personal de secretaría pertenece el autor desde hace diecisiete años. Destaca un apartado inicial titulado "El autor” (p. 11-12), en que se relata una pequeña biografía.

82 Pichincha 1093. Se informa en la portada que la editorial Grabo es editora de El Ajedrez Americano.

${ }^{83}$ De los 24 diagramas, 10 ilustran las historias y los otros 14 son los problemas relacionados con los finales artísticos.

${ }^{84}$ En el caso de los problemas, son literarios, aunque se puede aplicar al tablero de ajedrez.

${ }^{85}$ Carretas, 14, Madrid. En la cubierta posterior indica el precio de venta: cinco pesetas.

${ }^{86} 103$ diagramas hacen referencia a los problemas incluidos en la obra de 1283, Libro de Ajedrez, Dados y Tablas. Aparece en la obra la foto del autor y un grabado en la cubierta del rey Don Alfonso El Sabio con un escribano.

${ }^{87}$ La obra, no localizada, es citada en Els Escacs a Catalunya (Bibliografía, 1930).

${ }^{88}$ Se indica en la portada que "La fuerza moral de la F.I.D.E. reside en la disciplina de las unidades afiliadas". Se vende en la Federación Española de Ajedrez, en la Rambla Cataluña, 86.

89 El autor, que fue campeón argentino, fue el director de la revista El ajedrez Americano -el n. ${ }^{\circ} 1$ aparece en octubre de $1927-$.

${ }^{90}$ Conjunto de 4 vol. (1930-1943), aunque los tres últimos posteriores a 1939 (II. Estrategia, III. Conformaciones de peones, IV. Estrategia Superior). La obra se incluye en Semilla de ajedrez (1933) y en CEBA [núm. 32], por lo tanto, era conocida en España. Pero además es una obra de gran repercusión que tiene numerosas ediciones y reproducciones y citada en multitud de libros y publicaciones: Curso superior de ajedrez, de Reti, en un artículo del Dr. Rey Ardid, en La Vanguardia con el título de "Bibliografía de ajedrecista", dentro de la Sección de Ajedrez (20 de enero de 1933, p. 1). En este artículo Ardid explica que quizá el tomo II no aparezca nunca. Se comprueba que entre la publicación del tomo I y de los otros tomos, pasó más de una década.

${ }^{91}$ Grabo es la editora de la revista El Ajedrez Americano, ubicada en la calle Estados Unidos, 1408 de Buenos Aires.

92 Otras ediciones son de la editorial Sopena Argentina (1942, 1952, 1958, 1959, 1972, 1982, 1990). Además, la Casa del Ajedrez de Madrid (2000 y 2006) y la editorial Colihue de Buenos Aires (2005), han hecho reproducciones.

${ }_{93}$ Foto del autor.

${ }^{94}$ Ubicada en la calle Moreno, 500 esq. Bolívar. Impreso en Tall. Gráf. “Damiano”, de Zes y Tejero, en Corrientes, 439. El ejemplar se vendía en la librería Sintes (Índice Bibliográfico, 1933) y la $L u z$ anunció la obra (Ajedrez, 1932).

${ }^{95}$ La primera edición es de 1925 tiene 75 p. y 23 cm. Hay otras ediciones, como en 1948 de la ed. Grabo.

96 Aparece un grabado de Ricardo Reti por M. Gianolio. En la parte posterior de la cubierta anterior se anuncia la tienda de artículos "Cazaban \& Fernández”. En la cubierta posterior se anuncia el Club Argentino de Ajedrez, la obra de Grau Tratado General de Ajedrez, de la ed. Grabo, y el Club de Ajedrez Jaque Mate. También se anuncian libros de ajedrez, con su precio de venta en Buenos Aires. Entre las obras, se encuentra el Manual de Paluzíe.

${ }^{97}$ El autor de este artículo posee un ejemplar firmado a mano por el traductor en fecha de octubre de 1930.

${ }^{98}$ El traductor es León Colmán Lerner, que también adapta la $2^{\mathrm{a}}$ edición del Curso Superior de Ajedrez, de Ricardo Reti, tal y como se anuncia en la obra.

${ }^{99}$ En un ejemplar que posee el autor de este artículo destaca una hoja publicitaria suelta anunciando: Concurso. Para lectores del Suplemento del Curso Superior de Ajedrez.

${ }^{100}$ Calle Larra, 13. Precio de venta: 3 ptas.

${ }^{101}$ Hay 9 páginas estan numeradas en números romanos.

${ }^{102}$ La obra es citada por Sánchez (1933).

103 En la cubierta anterior se detallan artículos en venta de la compañía Argentina de Billares. En la cubierta posterior se detallan, con una pequeña síntesis, diferentes publicaciones de ajedrez de la editorial Grabo: La revista El Ajedrez Americano, el Tratado General de Ajedrez i Cartilla de Ajedrez, de Grau; Combinaciones y Celadas en las aperturas, de Palau; Sugestiones para la estrategia ajedrecistica, de Tartakower; Trebejos de Viaña.

${ }^{104}$ Fue director de la La Stratégie (1908-1940).

${ }^{105}$ Título original: Manual des Echecs -ed. La Stratégie- (1917).

106 Aribau, 175-177. El impresor es Ponsa, ubicado en Iradier, 5A de Barcelona. 
107 Hay posteriores ediciones (1935, 1943, 1946 -reimpresión-, 1950).

108 Hay dos páginas finales de anuncios de dos obras de la editorial.

109 La obra se cita en Semilla de Ajedrez (1933), por lo tanto era conocida en España. Además, en el ejemplar, propiedad del autor, si indica mediante un adhesivo, la Librería Nacional y Extranjera de Barcelona (Rambla Cataluña, 72) como punto de venta.

110 También se dice de esta obra que es “Editora de 'El Ajedrez Americano'. Estados Unidos, 1408”.

111 Otras ediciones (ed. Sopena Argentina; Buenos Aires): 1940, 1945, 1952, 1967, 1974, 1982, 1990. Hay una edición de 1961 de la editorial Orbe (la Habana). También ha reproducido la obra: Limusa/Noriega editores, en 1995 (Méjico).

112 Se incluyen en las 88 p., un apartado de soluciones y el índice.

113 En el ejemplar, proveniente de la colección de Paluzíe, de la Biblioteca de Cataluña, hay una dedicatoria a mano 'A Don Juan Paluzíe Lucena, nuestro Decano, con todo cariño y respeto’ de Juan B. Sánchez Pérez. Madrid, mayo 1931.

114 Rusia.

115 También se dice de la editorial que es: "Editora de 'El Ajedrez Americano'. Calle Estados Unidos 1408".

116 De esta obra se han encontrado más ediciones de la editorial Sopena Argentina (Buenos Aires) y traducido directamente del alemán por Carlos O. Müller (1941, 1946, 1954, 1960, 1967, 1973 -7ª edición-, 1978 -9ª edición-).

${ }^{117}$ Al final de la obra hay, además del índice, un índice de partidas y en las dos últimas páginas (no numeradas) publicaciones de la editorial: cinco libros (Tratado General de Ajedrez, Combinaciones y Celadas en las Aperturas, Reglamento y Código del Juego del Ajedrez, "Trebejos" y Combinaciones Brillantes) y una revista (Ajedrez Americano). De cada publicación se hace un pequeño resumen. En la cubierta posterior se indica el precio: $\$ 2,50$.

${ }^{118}$ Foto del autor (p. 5).

119 P. 7-8. Resultados obtenidos por el autor desde 1906 hasta 1930 en los diferentes torneos (se especifica el torneo, colocación, partidas ganadas, partidas perdidas, partidas tablas y puntos).

120 Checoslovaquia.

121 Título original de la obra: Die Meister des Schachbretts (1930). En 2010, Jens-Erik Rudolph Verlag ha publicado el libro (ed.: Books on Demand GmbH, Norderstedt. Hamburgo). Hay un prólogo del traductor donde se comenta que es una obra póstuma y que la segunda parte se publicará al cabo de un tiempo. De todas formas, solo hemos encontrado la segunda parte en la editorial Grabo, Buenos Aires, 1948 (esta editorial publicó en 1940 la primera parte. El traductor es Bort-Barbosa) y posteriormente por Ricardo Aguilera en la década de los sesenta. Precio de venta 10 ptas., según la referencia de la obra que se hace en Luz (Ajedrez, 1932). Este mismo precio se indica en el anuncio de la Librería Nacional y Extranjera aparecido en Reus Torneig Internacional d'Escacs (1936).

122 Rambla Cataluña, 72. Se detalla en la contraportada: Imp. Luis Guinart Peix. Barcelona.

123 Se han hecho diferentes traducciones y copias de la obra: ed. Grabo, Buenos Aires, 1940 (en 1948 esta editorial publicó la segunda parte); ed. Fundamentos y Ricardo Aguilera (Madrid): 1965, 1968, 1972, 1982, 1987, 1997, 2006. También ha publicado la obra: Editoriales de Derecho Reunidas (1997).

124 Hay ocho páginas iniciales de introducción en números romanos. Foto del autor que se encuentra en la página anterior a la portada.

125 Aribau, 175 y 177, Barcelona. El nombre completo de la editorial es Bartolomé Bauzá.

${ }^{126}$ En Garzón, Alió y Artigas (2012) se comenta que hay una segunda edición c. 1936 que tuvo diferentes reimpresiones (c. 1945, c. 1950).

${ }^{127}$ Hay dos páginas finales de anuncios de la editorial B. Bauzá.

128 Luisa Fernanda, 5

129 Luisa Fernanda, 5.

130 En el prólogo. Creemos importante destacar dos fotos que hacen referencia a niños jugando en la "Escuela del Mar” (Barcelona). En este centro, se dedica a "este deporte especial atención” (p. 8). En la página 59 aparece una tabla que relaciona el nombre de cada pieza en diferentes idiomas

131 Se incluye la novela simbólica: Don Sandalio, jugador de ajedrez.

132 Impresión y encuadernación de Julián Espinosa (Augusto Figueroa, 4, Madrid). En la cubierta posterior se indica que el ejemplar se vendía en la Librería Española y Extranjera Beltrán (Príncipe, 16, Madrid). La obra además se vendía en la librería Sintes, como se comprueba en el Índice Bibliográfico (1933)

${ }^{133}$ El reglamento de ajedrez comprende las páginas 5 a la 15. Se encuentran dos ejemplares con diferentes cubiertas. En uno de los dos hay una imagen de cinco naipes españoles y precio de venta de dos pesetas.

134 Dibujo en la portada de J. Galiay. Contraportada: Precio: 12,5 ptas. Concesionario exclusivo para la venta: Librería Gasca, Coso, 31, Zaragoza.

135 F. Martínez, Audiencia, 3 y 5.

136 Se incluye el índice alfabético de jugadores en las páginas 265 y 266. Hay una última página de índice de aperturas.

137 Luisa Fernanda, 5.

138 Berbera editores S.A. de C.V. (Méjico) ha hecho una copia de la obra (2009 y 156 p.).

139 Hay una última página con las obras del autor y su precio. El libro citado tiene un precio en rústica de 18 pesetas y encuadernado de 20 pesetas (en la cubierta posterior de la obra se indica también el precio). En esta página también se nombra la librería Blasco Ibañez, 39, Madrid. Aparecen la fotos de: Lasker-Rubinstein en un ensayo de partida (p. 21), partida de ajedrez viviente (p. 22), autómata Torres Quevedo (p. 85), Alekhine (p. 109), Capablanca (p. 109), Rey Ardid (p. 110).

${ }^{140}$ Original publicado en 1931 en Francia (76 p.; $\left.18 \mathrm{~cm}\right)$.

141 Aribau, 175 y 177, Barcelona. Gráfica Industrial: Enrique Granados, 70, Barcelona.

142 Se encuentra una segunda edición (c. 1945) y una segunda reimpresión de ésta. La obra se tradujo al inglés en 1931 por Philip Hereford y en 1937 por G.E. Smith i Dasch. Además la obra tuvo repercusión en Sudamérica, publicándose en 1944 (ed. Grabo en Buenos Aires. 88 p.; 16 cm), 1954 (por la misma editorial con 49 p. y $21 \mathrm{~cm}$ y en la que se comenta que la traducción es directa del original francés por J. M. Suárez) y una quinta edición en 1958, por la misma editorial.

${ }^{143}$ Hay una página final anunciando otro libro de la editorial.

144 Se comenta que es la editora de "El Ajedrez Americano". 
145 Otras ediciones: 1938 (3a ed.), 1942 (4a ed.), 1946 (5a ed.), 1954 (6a ed.) 1961 (7a ed.). Podría ser que la primera edición fuese de 1929, pero no se ha hallado ningún ejemplar.

${ }^{146}$ Los dos dibujos son de cada pieza de ajedrez. Hay otro dibujo en la cubierta anterior (tablero ajedrez con un peón, un caballo y un alfil). La tabla hace referencia a los emparejamientos para torneos.

${ }^{147}$ Rambla Cataluña, 72. Se indica Imp. L. Guinart Peix, ubicada en Milá y Fontanals, 41. La obra se vendía al precio de 4 ptas. en la Librería Nacional y Extranjera, como se comprueba en el anuncio aparecido en la publicación Reus Torneig Internacional d'Escacs (1936).

148 La portada incluye el escudo del Club d'Escacs Barcelona.

149 Carrer Ample, 33. Se incluyen teléfonos: “12 456 y 11 147”. Se vende en la librería Catalonia, Rda. Sant Pere, 3 , por 7 pesetas.

150 Hay un índice final.

151 Seudónimo del que fue general de brigada Emilio Mola Vidal (Garzón, Alió y Artigas, 2012).

152 Tipografía Mergenthaler, Madrid.

153 Original de 1935, Lib. Bergua. Imp. Sáez Hermanos, Madrid. Otras ediciones: Ibéricas (Barcelona, 1939) / Imp. de Sáez Hermanos, 1940 (2a ed.), 1944 (3a ed.). La obra en 1935 tenía un coste de 2 ptas. y en 1939 de 2,5 ptas. La filosofía de la editorial era vender libros a bajo precio.

154 Se incluye posteriormente una errata y también una última hoja con el índice.

155 La obra la forman dos tomos, publicados el mismo año.

156 Pozas, 14. Teléfono 13 713. Se indica también en la portada de los dos tomos "Publicaciones de las escuelas de estudios árabes de Madrid y Granada. Serie A. Núm. 3”.

${ }^{157}$ La obra se terminó de imprimir el 26 de abril de 1935, con una tirada de quinientos ejemplares. La editorial Maxtor ha hecho un facsímil de la obra.

158 El autor es el hermano de Juan Bautista Sánchez Pérez, que escribe diferentes obras relacionadas con el ajedrez.

159 La obra pertenece a la colección Biblioteca de la Cultura Española (Director: Francisco Vera). Se indica también la dirección de M. Aguilar Editor: Marqués de Urquijo, 43. Apartado 8011. Madrid. También se indica: Bolafios y Aguilar (S. L.). Talleres gráficos. Altamirano, 50.

160 La obra se publicó entre diciembre de 1934 y febrero de 1935. Se han tenido en cuenta las informaciones y obras descritas en el pliegue de la cubierta posterior del libro propietario del autor.

161 El número de ilustraciones se refiere al capítulo del libro titulado "Libro de los juegos” (p. 229-242). Las noticias de dicho capítulo han sido tomadas del hermano del autor, concretamente de la obra El Ajedrez de Don Alfonso el Sabio.

162 Castelar, 7 (Reus). Teléfono 704.

163 Páginas no numeradas. Grabado en la cubierta de A. Dufresne, correspondiente al logotipo del torneo (piezas de ajedrez e iglesia de Sant Pere de Reus). En relación con el logotipo, se editaron también sellos. Diagrama relacionado con la ciudad de Reus, su entorno y ajedrecistas y torneos. Además se incluyen numerosos anuncios publicitarios. $22 \mathrm{~cm}$ de largo.

164 Editor: Carl Seither. Barcelona. Rambla Catalunya, 72. Imprenta Altés (Tuset, 28. Barcelona). Colección: Biblioteca de Ajedrez 2. Precio de venta: 3,5 ptas. Este precio se comprueba también en el anuncio de la Librería Nacional y Extranjera, aparecido en la obra de Reus Torneig Internacional d'Escacs (1936). La primera obra de esta colección es el Torneo Internacional de Sitges 1934.

165 En el mismo 1936 se hizo una reimpresión de la obra. (NEBEA, 2012).

166 Dibujo en la portada de Chacón XXXVIII. Contraportada: Librería General. Independencia, 8. Zaragoza. Precio: 8 pesetas. La obra incluye el reglamento de la FIDE de 1928.

${ }^{167}$ F. Martínez,Audiencia, 5.

168 Nota debajo del año: “Año de la victoria”. Otras ediciones del libro: 1939, 1942, 1943, 1951, 1962 , 1970 (1989 por la editorial Aguilera, Madrid).

${ }^{169}$ Hay una última página con la "Fe de erratas".

\section{BIBLIOGRAFÍA}

AGUIRRE, M. El Raja-Yoga. Barcelona: Imp. Clarasó, 1928.

Ajedrez. Luz, 4 de noviembre de 1932, n 260, p. 14.

ALEKHINE, A. Mis mejores partidas de ajedrez 1908-1923. Montevideo: Talleres Gráficos J. Florensa, 1929.

ARMENGOL, F. El Club d'Escacs Barcelona vist per mi. Barcelona: Pereda Impressor, 1935.

Bibliografía. Escacs a Catalunya, 1930, nº 37, p. 534.

BIZKAYA, L. Los juegos. Madrid: Aleu, 1910.

BLANCO, R. Bibliografia general de la educación física: con datos copiosos referentes a la biología, antropología, anatomía, fisiología, higiene, eugénica, puericultura, paidología, pedagogía y otras ciencias fundamentales del ejercicio corporal educativo. Madrid: Hernando, 1927.

BOLADO, G. Menéndez Pelayo y las verdades de la tradición. Eikasia, 2012, nº 7, p. 251-262.

BRASÓ, J. Bibliografía de ajedrez existente en España, de 1800 a 1939. Lecturas: Educación Física y Deportes 2013, vol. 18, $\mathrm{n}^{0}$ 184. Disponible en: <http://www.efdeportes.com/efd184/bibliografia-de-ajedrez-en-espana-18001939.htm> [Consulta: 7 de enero de 2016].

BRASÓ, J. Inicios del ajedrez en la prensa y en las publicaciones deportivas en España (1861-1915). Recorde: Revista de História do Esporte, 2014, vol. 7, nº 1, p. 1-34.

BRASÓ, J. Thomas Arnold, Pere Vergés i els jocs organitzats. Els escacs, un projecte educatiu a l'Escola del Mar (1922-1938), Temps d'Educació, 2015, nº 49, p. 11-30. 
BRASÓ, J. y TORREBADELLA, X. El joc del 'rescat' a Catalunya. Un projecte educatiu a l'Escola del Mar de Pere Vergés. Temps d'Educació, 2014, nº 47, p. 191-212.

BRUNET, J. El ajedrez. Investigaciones sobre su origen. Barcelona: Lib. l'Avenç, 1891.

CAPABLANCA, J.R. Cartilla de Ajedrez. La Habana: Imp. del Avisador Comercial, 1913.

CESSOLIS, J. De les costums dels homens e dels oficis dels nobles, sobre l Joch dels Escachs. Barcelona: L'Avenç, 1900.

CESSULIS, J. Libre de bones costumes dels homens e dels oficis dels nobles. Barcelona: Francisco X: Altés, 1902.

CLEMENTE. Crónica del Sport 1896, vol. 4, n 24, p. 1.

CLEMENTE VÁZQUEZ, A. Biblioteca "Andrés C. Vázquez". La Habana: Imp. Avisador Comercial, 1899.

CLEMENTE VÁZQUEZ, A. El ajedrez magistral. La Habana: Avisador Comercial, 1900.

COHN, H. Lances humorísticos. Guatemala: Unión Tipográfica, 1936.

De Ajedrez. Stadium IV, 1914, nº 70, p. 594.

DELAIRE, E. Manual de ajedrez. Barcelona: Bauzá, 1931.

DI FELICE, G. Chess Periodicals. An Annotated International Bibliography, 1836-2008. North Carolina: McFarland \& Company, 2010.

DÍEZ, I. Las bio-bibliografías: estado actual y metodología. Cuadernos de documentación multimedia, 2000, n 10 , p. 67-77.

DUPUY-MAZUEL, H. El jugador de ajedrez. Paris-Madrid: Ed. Españolas, 1927.

FE, F. Catálogo de las obras de fondo y algunas de surtido clasificadas por materias y orden alfabético de autores. Madrid: Librería Nacional y extranjera de Fernando Fe, 1903.

Federación Argentina de Ajedrez. Digesto de Ajedrez. Buenos Aires: Federación Argentina de Ajedrez, 1930.

Federación Internacional de Ajedrez. Reglamento de ajedrez de la Fédération Internationale des Echecs. Valencia: Imp. la Semana Gráfica, 1930.

GARCÍA, J. Llibre per aprendrer a jugar als escacs en 8 dies. Terrassa: Imp. La Industria, 1921.

GARZÓN, J.A.; ALIÓ, J. y ARTIGAS, M. NEBEA. Nuevo Ensayo de Bibliografía Española de Ajedrez. 1238-1938. Con apuntes para la historia del ajedrez español. Valencia: Romeditors, 2012.

GELABERT, J.A. y MARTÍNEZ, G. Aperturas de ajedrez. Buenos Aires: Grabo, 1930.

GRAU, G. Tratado general de ajedrez. Buenos Aires: Grabo, 1930.

GRAU, R. Cartilla de ajedrez. Buenos Aires: Grabo, 1931.

HOOPER, W. Manual práctico de ajedrez. Madrid: Ed. Ibéricas, 1935.

Índice Bibliográfico. Barcelona: Lib. Sintes, 1933.

Índice Bibliográfico de obras de fondo y algunas de surtido. Madrid: Francisco Beltrán, 1912.

KOLTANOWSKI, G. Torneo Internacional de Hastings. Barcelona: C. Seither, 1936.

LASKER, E. Curso de Ajedrez. Buenos Aires: Librería de la Vda. de C. Bouret, 1920.

LASKER, E. El sentido común en ajedrez. México: Andrés Botas e hijo, 1918.

LLAVERIAS, A. Catálogo de la Biblioteca del Gimnasio Colón. Barcelona: Antoni Palau y Dulcet, 1910.

LOBATO, J.M. El contragambito de Greco. México: Antonio Vázquez, 1914.

MARCOFF, A. El ajedrez. Barcelona: B. Bauzá, 1933.

MARTÍN, R. Manual del ajedrecista. Barcelona: Saurí Sabater, 1897.

MARTÍNEZ DE CARVAJAL, V. Algunas consideraciones sobre el ajedrez. El Museo Universal, 1865, n 25, p. 199.

MENÉNDEZ, M. De Re Bibliographica. Al señor D. Gumersindo Laverde Ruiz. Revista Europea 1876, vol. 8, $\mathrm{n}^{\circ} 125$, p. 65-73.

Moral del ajedrez. La Granja, Revista de Agricultura y Biblioteca Rural, Periódico de la Sociedad de agricultura del Ampurdán, 1853, nº 7, p. 115-118.

NIESSEL, A. Instrucción de los oficiales por medio del Juego de la Guerra. Barcelona: Toribio Taberner, 1908.

OLIVERA, J. Prólogo. En: TORREBADELLA FLIX, X. Repertorio bibliográfico e inédito de la educación física y el deporte en España (1800-1939). Madrid: Fundación Universitaria Española, 2011, p. 9-13.

PALAU, A. Manual del librero hispano-americano: inventario bibliográfico... Palacete Palau Dulcet: Barcelona, 1923-1945.

PALAU, L. Combinaciones y celadas en las aperturas. Buenos Aires: Grabo, 1929.

PALUZÍE, J. Manual de ajedrez. Tomo I. Barcelona: Hijos de Paluzíe, 1905.

PALUZÍE, J. Sesdek problemoj. Barcelona: Filoj de Paluzíe, 1909.

PALUZÍE, J. Primer ensayo de bibliografia española de ajedrez. Barcelona: Hijos de Paluzíe, 1912.

PALUZÍE, J. Un artista en ajedrez. Barcelona: Hijos de Paluzíe, 1913.

PALUZÍE, J. Primer libro del ajedrecista. Barcelona: Hijos de Paluzíe, 1917.

PALUZÍE, J. Manual de ajedrez. Parte sexta (última). Miscelánea. Barcelona: s.e., 1921.

PALUZÍE, J. Catálogo de la exposición bibliográfica de ajedrez. Barcelona: Casa Provincial de la Caridad, 1943.

PAREJA, F.M. Libro del ajedrez, de sus problemas y sutilezas, de autor árabe desconocido. Madrid: Imp. de Estanislao Maestre, 1935. 
PASTOR, J.L. La Educación Física en España: fuentes bibliográficas básicas. Alcalá de Henares: Universidad de Alcalá, 1995.

PÉREZ, J. El ajedrez en la Argentina. Buenos Aires: Imp. Tixi y Schaffner, 1920.

PIN y SOLER, J. Reglas Morals y de bona criansa. Barcelona: Estampa de Henrich i Comp., 1892.

POE, E.A. Nuevas historias extraordinarias. Madrid: Matev, 1918.

PONS, F. Ensayo bio-bibliográfico sobre los Historiadores y Geógrafos arábigo-españoles. Madrid: Est. Tip. de San Francisco de Sales, 1898.

Primer torneig internacional d'escacs. Barcelona: Generalitat de Catalunya, 1934.

Prontuario del juego del ajedrez. Sevilla: El Correo de Andalucía, 1900.

PUIG, E. Torneo de ajedrez para el campeonato de Barcelona. Barcelona: Hijos de Paluzíe, 1913.

PUIG, E. Apuntes críticos sobre la obra 150 fins de partie de Mr. henri Rinck. Barcelona: Bobes, 1908.

PUIG, E. La doctrina del final artístico. Barcelona: Tip. la Académica, 1923.

PUNZANO, V. Pensamiento bibliográfico de Menéndez Pelayo. I Simposium Internacional de Literatura Hispanoamericana. Santander: UIMP, 1981, p. 147-164.

Reglamentos admitidos por el uso, para los juegos de ajedrez, barracat, julepe, monte, mus, poker, ruleta, siete y media, trenta y cuarenta, tresillo, tute. Madrid: Biblioteca El defensor de Madrid, 1933.

RETI, R. Curso superior de ajedrez. Buenos Aires: Lib. de A. Garcia Santos, 1930a.

RETI, R. Suplemento al Curso Superior de Ajedrez. Buenos Aires: A. Garcia Santos, $1930 \mathrm{~b}$.

RETI, R. Los grandes maestros del tablero. Barcelona: Seither, 1932.

Reus. Torneig Internacional d'escacs. Reus: Imp. Cuscó, 1936.

REY, Dr. Sección de Ajedrez. Bibliografía Ajedrecística. La Vanguardia, 20 de enero de 1933, p. 12.

REY, R. Cien partidas de ajedrez. Zaragoza: La Académica, 1934.

REY, R. Los principios del ajedrez. Zaragoza: La Académica, 1939.

RINCK, H. 300 fins partie. Barcelona: Hijos de Paluzíe, 1919.

RINCK, H. 700 Fins de Partie. Barcelona: la Académica, 1927.

RODRÍGUEZ, L. Ajedrez. Torneo de aficionados en San Sebastián. Madrid: Imp. del Asilo de Huérfanos, 1912.

ROUSSELL-PARCY, J. ¿Quiere V. jugar al ajedrez? Barcelona: B. Bauzá, 1914.

SÁNCHEZ, J.B. Semilla de Ajedrez. Madrid: Imp. Cleto Vallinas, 1933.

SÁNCHEZ, J.B. Alfonso X El Sabio. Madrid: M. Aguilar, c. 1934-1935.

SÁNCHEZ, J.B. Diccionario ilustrado de ajedrez. Madrid: Imp. Cleto Vallinas, 1934.

SÁNCHEZ, J.B. El ajedrez de D. Alfonso el Sabio. Madrid: Franco Española, 1929.

SÁNCHEZ, J.B. Epitome de finales y problemas. Madrid: Prensa Moderna, 1931.

SÁNCHEZ, J.B. Nómina de aperturas. Madrid: Prensa Moderna, 1930.

SÁNCHEZ, J.B. Una clasificación de aperturas de ajedrez. Madrid: Imp. Cleto Vallinas, 1933.

SÁNCHEZ-OCAÑA, J. Barcelona, paraíso de los jugadores de ajedrez. Cuarenta clubs; veinte mil aficionados... Estampa, 1928, no 371, p. 11-14.

SANDOVAL, A. Colección de los problemas de ajedrez. México: Compañía ed. Católica, 1906.

SOLALINDE, A. Alfonso X El Sabio. Madrid: Jiménez-Fraud, 1922.

TARTAKOWER, S. Sugestiones para la estrategia ajedrecística. Buenos Aires: Grabo, 1931.

TOLOSA, J. Problemas de ajedrez. Barcelona: Hijos de Paluzíe, 1917.

Torneo internacional de Sitges año 1934. Barcelona: C. Seither, 1935.

TORREBADELLA, X. Rufino Blanco Sánchez y las fuentes bibliográficas de la educación física y el deporte en España. RICYDE. Revista Internacional de Ciencias del Deporte, 2014, vol. 10, nº 37, p. 281-284.

TORREBADELLA, X. y NOMDEDEU, A. Repertorio bibliográfico de futbol en España (1900-1936). 121 obras para interpretar el impacto social del fútbol en la historia contemporánea. Apunts. Educación Física y Deportes, 2014, $n^{\circ} 115$, p. 7-32.

TORREBADELLA, X. y OLIVERA, J. Las cien obras clave del repertorio bibliográfico español de la educación física y el deporte en su proceso de legitimación e institucionalización. Revista General de Información y Documentación, 2012, n 22, p. 119-168.

UNAMUNO, M. San Manuel Bueno, Mártir y tres historias más. Madrid: Biblioteca El defensor, 1933.

VIAÑA, M. Trebejos. Buenos Aires: Grabo, 1929.

VILLAESPESA, F. Era él. Poema en un acto. Madrid: Sociedad de Autores Españoles, 1916.

WILLEMANNS. ¿Quiere usted aprender a jugar al ajedrez? Barcelona: F. Granada y Cie, 1909.

ZNOSKO-BOROVSKY, E. Cómo no debe jugarse al ajedrez. Barcelona: Bauzá, 1934. 